J. DIFFERENTIAL GEOMETRY

93 (2013) 269-297

\title{
MINIMAL MODELS, FORMALITY, AND HARD LEFSCHETZ PROPERTIES OF SOLVMANIFOLDS WITH LOCAL SYSTEMS
}

\author{
HisASHI KASUYA
}

\begin{abstract}
For a simply connected solvable Lie group $G$ with a cocompact discrete subgroup $\Gamma$, we consider the space of differential forms on the solvmanifold $G / \Gamma$ with values in a certain flat bundle so that this space has a structure of a differential graded algebra (DGA). We construct Sullivan's minimal model of this DGA. This result is an extension of Nomizu's theorem for ordinary coefficients in the nilpotent case. By using this result, we refine Hasegawa's result of formality of nilmanifolds and Benson-Gordon's result of hard Lefschetz properties of nilmanifolds.
\end{abstract}

\section{Introduction}

The main purpose of this paper is to compute the de Rham cohomology of solvmanifolds with values in local coefficients associated to some diagonal representations by using the invariant forms and the unipotent hulls. The computations are natural extensions of Nomizu's computations of untwisted de Rham cohomology of nilmanifolds by the invariant forms in [23]. The computations give natural extensions of Hasegawa's result of formality of nilmanifolds ([15]) and Benson and Gordon's result of hard Lefschetz properties of nilmanifolds $([\mathbf{6}])$.

First we explain the central tools of this paper, called the unipotent hulls and algebraic hulls. Let $G$ be a simply connected solvable Lie group. There exists a unique algebraic group $\mathbf{H}_{G}$ called the algebraic hull of $G$ with an injection $\psi: G \rightarrow \mathbf{H}_{G}$ so that:

1) $\psi(G)$ is Zariski-dense in $\mathbf{H}_{G}$;

2) the centralizer $Z_{\mathbf{H}_{G}}\left(\mathbf{U}\left(\mathbf{H}_{G}\right)\right)$ of $\mathbf{U}\left(\mathbf{H}_{G}\right)$ is contained in $\mathbf{U}\left(\mathbf{H}_{G}\right)$;

3) $\operatorname{dim} \mathbf{U}\left(\mathbf{H}_{G}\right)=\operatorname{dim} G$;

where we denote $\mathbf{U}(\mathbf{H})$ the unipotent radical of an algebraic group $\mathbf{H}$. We denote $\mathbf{U}_{G}=\mathbf{U}\left(\mathbf{H}_{G}\right)$ and call it the unipotent hull of $G$.

We consider Hain's DGAs in [14] which are expected to be effective techniques for studying de Rham homotopy theory of non-nilpotent

Received 8/11/2011. 
spaces. Let $M$ be a $C^{\infty}$-manifold, $\rho: \pi_{1}(M, x) \rightarrow\left(\mathbb{C}^{*}\right)^{n}$ a representation; and $\mathbf{T}$ the Zariski-closure of $\rho\left(\pi_{1}(M, x)\right)$ in $\left(\mathbb{C}^{*}\right)^{n}$. Let $\left\{V_{\alpha}\right\}$ be the set of one-dimensional representations for all characters $\alpha$ of $\mathbf{T}$, let $\left(E_{\alpha}, D_{\alpha}\right)$ be a rank one flat bundle with the monodromy $\alpha \circ \rho$ and with $A^{*}\left(M, E_{\alpha}\right)$ the space of $E_{\rho}$-valued $C^{\infty}$-differential forms. Denote $A^{*}\left(M, \mathcal{O}_{\rho}\right)=\bigoplus_{\alpha} A^{*}\left(M, E_{\alpha}\right)$ and $D=\bigoplus_{\alpha} D_{\alpha}$. Then $\left(A^{*}\left(M, \mathcal{O}_{\rho}\right), D\right)$ is a cohomologically connected (i.e. the 0 -th cohomology is isomorphic to the ground field) DGA. In this paper we construct Sullivan's minimal model ([29]) of such DGAs on solvmanifolds.

On simply connected solvable Lie groups, we consider DGAs of leftinvariant differential forms with local systems which are analogues of Hain's DGAs. Suppose $G$ is a simply connected solvable Lie group and $\mathfrak{g}$ is the Lie algebra of $G$. Consider the adjoint representation Ad : $G \rightarrow$ $\operatorname{Aut}(\mathfrak{g})$ and its derivation ad $: \mathfrak{g} \rightarrow D(\mathfrak{g})$, where $D(\mathfrak{g})$ is the Lie algebra of the derivations of $\mathfrak{g}$. We construct representations of $\mathfrak{g}$ and $G$ as follows.

Construction 1.1. Let $\mathfrak{n}$ be the nilradical of $\mathfrak{g}$. There exists a subvector space (not necessarily Lie algebra) $V$ of $\mathfrak{g}$ so that $\mathfrak{g}=V \oplus \mathfrak{n}$ as the direct sum of vector spaces and for any $A, B \in V\left(\operatorname{ad}_{A}\right)_{s}(B)=0$ where $\left(a d_{A}\right)_{s}$ is the semi-simple part of $\operatorname{ad}_{A}$ (see [12, Proposition III.1.1]). We define the map $\operatorname{ad}_{s}: \mathfrak{g} \rightarrow D(\mathfrak{g})$ as $\operatorname{ad}_{s A+X}=\left(\operatorname{ad}_{A}\right)_{s}$ for $A \in V$ and $X \in \mathfrak{n}$. Then we have $\left[\operatorname{ad}_{s}(\mathfrak{g}), \operatorname{ad}_{s}(\mathfrak{g})\right]=0$ and $\operatorname{ad}_{s}$ is linear (see $[\mathbf{1 2}$, Proposition III.1.1]). Since we have $[\mathfrak{g}, \mathfrak{g}] \subset \mathfrak{n}$, the map $\operatorname{ad}_{s}: \mathfrak{g} \rightarrow D(\mathfrak{g})$ is a representation and the image $\operatorname{ad}_{s}(\mathfrak{g})$ is abelian and consists of semisimple elements. We denote by $\operatorname{Ad}_{s}: G \rightarrow \operatorname{Aut}(\mathfrak{g})$ the extension of $\operatorname{ad}_{s}$. Then $\operatorname{Ad}_{s}(G)$ is diagonalizable.

Let $\mathbf{T}$ be the Zariski-closure of $\operatorname{Ad}_{s}(G)$ in $\operatorname{Aut}\left(\mathfrak{g}_{\mathbb{C}}\right)$. Then $\mathbf{T}$ is diagonalizable. Let $\left\{V_{\alpha}\right\}$ be the set of one-dimensional representations for all characters $\alpha$ of $\mathbf{T}$. We consider $V_{\alpha}$ the representation of $\mathfrak{g}$ which is the derivation of $\alpha \circ \mathrm{Ad}_{s}$. Then we have the cochain complex of Lie algebra $\left(\bigwedge \mathfrak{g}_{\mathbb{C}}^{*} \otimes V_{\alpha}, d_{\alpha}\right)$. Denote $A^{*}\left(\mathfrak{g}_{\mathbb{C}}, \operatorname{ad}_{s}\right)=\bigoplus_{\alpha} \wedge \mathfrak{g}_{\mathbb{C}}^{*} \otimes V_{\alpha}$ and $d=\bigoplus_{\alpha} d_{\alpha}$. Then $\left(A^{*}\left(\mathfrak{g}_{\mathbb{C}}, \operatorname{ad}_{s}\right), d\right)$ is a cohomologically connected DGA. In this paper we compute the cohomology of this DGA by the unipotent hull $\mathbf{U}_{G}$ of $G$. Let $\mathfrak{u}$ be the Lie algebra of $\mathbf{U}_{G}$ and $\bigwedge \mathfrak{u}^{*}$ be the cochain complex of the dual space $\mathfrak{u}^{*}$ of $\mathfrak{u}$. We prove the following theorem.

Theorem 1.1 (Theorem 5.4). We have a quasi-isomorphism (i.e. a morphism which induces a cohomology isomorphism) of DGAs

$$
\bigwedge \mathfrak{u}^{*} \rightarrow A^{*}\left(\mathfrak{g}_{\mathbb{C}}, \operatorname{ad}_{s}\right) .
$$

Thus $\bigwedge \mathfrak{u}^{*}$ is Sullivan's minimal model of $A^{*}\left(\mathfrak{g}_{\mathbb{C}}, \operatorname{ad}_{s}\right)$.

Suppose $G$ has a lattice $\Gamma$, i.e. a cocompact discrete subgroup of $G$. We call a compact homogeneous space $G / \Gamma$ a solvmanifold. We have $\pi_{1}(G / \Gamma) \cong \Gamma$. For the restriction of the semi-simple part of the adjoint 
representation $\operatorname{Ad}_{\left.s\right|_{\Gamma}}$ on $\Gamma$, we consider Hain's DGA $A^{*}\left(G / \Gamma, \mathcal{O}_{\operatorname{Ad}_{s_{\mid}}}\right)$. By using Theorem 1.1, we prove:

Theorem 1.2 (Corollary 7.5). Let $G$ be a simply connected solvable Lie group with a lattice $\Gamma$, and let $\mathbf{U}_{G}$ be the unipotent hull of $G$. Let $\mathfrak{u}$ be the Lie algebra of $\mathbf{U}_{G}$. Then we have a quasi-isomorphism

$$
\bigwedge \mathfrak{u}^{*} \rightarrow A^{*}\left(G / \Gamma, \mathcal{O}_{\operatorname{Ad}_{s \mid}}\right) \text {. }
$$

Thus $\bigwedge \mathfrak{u}^{*}$ is Sullivan's minimal model of $A^{*}\left(G / \Gamma, \mathcal{O}_{\operatorname{Ad}_{s \mid}}\right)$.

If $G$ is nilpotent, the adjoint operator Ad is a unipotent representation and hence $A^{*}\left(G / \Gamma, \mathcal{O}_{\operatorname{Ad}_{s \mid \Gamma}}\right)=A_{\mathbb{C}}^{*}(G / \Gamma)$ and $A^{*}\left(\mathfrak{g}_{\mathbb{C}}, \operatorname{ad}_{s}\right)=\bigwedge \mathfrak{g}_{\mathbb{C}}=$ $\bigwedge \mathfrak{u}^{*}$. In this case, Theorem 1.2 reduces to the classical theorem proved by Nomizu in [23]. Moreover, this result gives more refined computations of untwisted de Rham cohomology of solvmanifolds than the results of Mostow and Hattori (see Corollary 7.4 and Section 10).

We call a DGA $A$ formal if there exists a finite diagram of morphisms

$$
A \rightarrow C_{1} \leftarrow C_{2} \cdots \leftarrow H^{*}(A)
$$

such that all morphisms are quasi-isomorphisms; and we call manifolds $M$ formal if the de Rham complex $A^{*}(M)$ is formal. In [15] Hasegawa showed that formal nilmanifolds are tori. By the results of this paper, we have a natural extension of Hasegawa's theorem for solvmanifolds.

Theorem 1.3 (Theorem 8.2). Let $G$ be a simply connected solvable Lie group. Then the following conditions are equivalent:

(A) The DGA $A^{*}\left(\mathfrak{g}_{\mathbb{C}}, \operatorname{ad}_{s}\right)$ is formal.

(B) $\mathbf{U}_{G}$ is abelian.

(C) $G=\mathbb{R}^{n} \ltimes_{\phi} \mathbb{R}^{m}$ such that the action $\phi: \mathbb{R}^{n} \rightarrow \operatorname{Aut}\left(\mathbb{R}^{m}\right)$ is semisimple.

Moreover, suppose $G$ has a lattice $\Gamma$. Then the above three conditions are equivalent to the following condition:

(D) $A^{*}\left(G / \Gamma, \mathcal{O}_{\operatorname{Ad}_{s \mid \Gamma}}\right)$ is formal.

In [6] Benson and Gordon showed that symplectic nilmanifolds with the hard Lefschetz properties are tori. We can also have an extension of Benson and Gordon's theorem.

Theorem 1.4 (Theorem 8.4). Let $G$ be a simply connected solvable Lie group. Suppose $\operatorname{dim} G=2 n$ and $G$ has an $G$-invariant symplectic form $\omega$. Then the following conditions are equivalent:

$$
[\omega]^{n-i} \wedge: H^{i}\left(A^{*}\left(\mathfrak{g}_{\mathbb{C}}, \operatorname{ad}_{s}\right)\right) \rightarrow H^{2 n-i}\left(A^{*}\left(\mathfrak{g}_{\mathbb{C}}, \operatorname{ad}_{s}\right)\right)
$$

is an isomorphic for any $i \leq n$.

(B) $\mathbf{U}_{G}$ is abelian.

(C) $G=\mathbb{R}^{n} \ltimes_{\phi} \mathbb{R}^{m}$ such that the action $\phi: \mathbb{R}^{n} \rightarrow \operatorname{Aut}\left(\mathbb{R}^{m}\right)$ is semisimple. 
Suppose $G$ has a lattice $\Gamma$ and $G / \Gamma$ has a symplectic form (not necessarily $G$-invariant) $\omega$. Then the conditions $(B)$ and $(C)$ are equivalent to the following condition:

$$
[\omega]^{n-i} \wedge: H^{i}\left(A^{*}\left(G / \Gamma, \mathcal{O}_{\operatorname{Ad}_{\left.s\right|_{\Gamma}}}\right)\right) \rightarrow H^{2 n-i}\left(A^{*}\left(G / \Gamma, \mathcal{O}_{\operatorname{Ad}_{\left.s\right|_{\Gamma}}}\right)\right)
$$

is an isomorphism for any $i \leq n$.

Remark 1.1. As a representation in an algebraic group, $\mathrm{Ad}_{s}$ is independent of the choice of a subvector space $V$ in Construction 1.1 (see Lemma 2.5). By this, the structures of DGAs $A^{*}\left(\mathfrak{g}_{\mathbb{C}}, \operatorname{ad}_{s}\right)$ and $A^{*}\left(G / \Gamma, \mathcal{O}_{\operatorname{Ad}_{\left.s\right|_{\Gamma}}}\right)$ are independent of the choice of a subvector space $V$.

Finally we consider relations with Kähler geometries. We review studies of Kähler structures on solvmanifolds briefly. See [5] and [17] for more details. In [7] Benson and Gordon conjectured that for a completely solvable simply connected Lie group $G$ with a lattice $\Gamma, G / \Gamma$ has a Kähler metric if and only if $G / \Gamma$ is a torus. In [16] Hasegawa studied Kähler structures on some classes of solvmanifolds which are not only the completely solvable type, and suggested a generalized version of Benson-Gordon's conjecture: A compact solvmanifold can have a Kahler structure if and only if it is a finite quotient of a complex torus that is a holomorphic fiber bundle over a complex torus with its fiber a complex torus. In [1] Arapura showed Benson-Gordon's conjecture and also showed that the fundamental group of a Kähler solvmanifold is virtually abelian by the result in $[\mathbf{2}]$. In [1] a proof of Hasegawa's conjecture was also written, but we notice that this proof contains a gap and Hasegawa complement in [17]. We also notice that Baues and Cortés showed a more generalized version of Benson-Gordon's conjecture for aspherical manifolds with polycyclic fundamental groups in [5].

By the theory of Higgs bundle studied by Simpson, we have a twisted analogue of formality (see $[\mathbf{1 1}]$ ) and the hard Lefschetz properties of compact Kähler manifolds. We have:

Theorem 1.5 (Special case of Thoerem 4.1). Suppose $M$ is a compact Kähler manifold with a Kähler form $\omega$, and that $\rho: \pi_{1}(M) \rightarrow\left(\mathbb{C}^{*}\right)^{n}$ is a representation. Then the following conditions hold:

(A) (formality) The $D G A A^{*}\left(M, \mathcal{O}_{\rho}\right)$ is formal.

(B) (hard Lefschetz) For any $0 \leq i \leq n$ the linear operator

$$
[\omega]^{n-i} \wedge: H^{i}\left(A^{*}\left(M, \mathcal{O}_{\rho}\right)\right) \rightarrow H^{2 n-i}\left(A^{*}\left(M, \mathcal{O}_{\rho}\right)\right)
$$

is an isomorphism where $\operatorname{dim}_{\mathbb{R}} M=2 n$.

Now by Theorem 1.5, formality and the hard Lefschetz property of DGA $A^{*}\left(G / \Gamma, \mathcal{O}_{\operatorname{Ad}_{\left.s\right|_{\Gamma}}}\right)$ are criteria for $G / \Gamma$ to have a Kähler metric. We will see such conditions are stronger than untwisted formality and the hard Lefschetz property. 
Remark 1.2. There exist examples of solvmanifolds $G / \Gamma$ which satisfy formality and the hard Lefschetz property of the untwisted de Rham complex $A^{*}(G / \Gamma)$, but do not satisfy formality and the hard Lefschetz property of $A^{*}\left(G / \Gamma, \mathcal{O}_{\operatorname{Ad}_{s \mid}}\right)$.

However, we will see that these criteria cannot classify Kähler solvmanifolds completely.

Remark 1.3. There exist examples of non-Kähler solvmanifolds which satisfy formality and the hard Lefschetz property of $A^{*}\left(G / \Gamma, \mathcal{O}_{\operatorname{Ad}_{s \mid} \Gamma}\right)$.

Acknowledgments. The author would like to express his gratitude to Toshitake Kohno for helpful suggestions and stimulating discussions. He would also like to thank Oliver Baues, Keizo Hasegawa, and Takumi Yamada for their active interest in this paper. This research is supported by JSPS Research Fellowships for Young Scientists. The author would like to express many thanks to the referee for his careful reading of the earlier version of manuscript with several important remarks, which lead to many improvements in the revised version.

\section{Preliminaries on algebraic hulls}

Let $G$ be a discrete group (resp. a Lie group). We call a map $\rho: G \rightarrow$ $G L_{n}(\mathbb{C})$ a representation, if $\rho$ is a homomorphism of groups (resp. Lie groups).

2.1. Algebraic groups. In this paper an algebraic group means an affine algebraic variety $\mathbf{G}$ over $\mathbb{C}$ with a group structure such that the multiplication and inverse are morphisms of varieties. All algebraic groups in this paper arise as Zariski-closed subgroups of $G L_{n}(\mathbb{C})$. Let $k$ be a subfield of $\mathbb{C}$. We call $\mathbf{G} k$-algebraic if $\mathbf{G}$ is defined by polynomials with coefficient in $k$. We denote as $\mathbf{G}(k)$ the $k$-points of $\mathbf{G}$. We say that an algebraic group is diagonalizable if it is isomorphic to a closed subgroup of $\left(\mathbb{C}^{*}\right)^{n}$ for some $n$.

2.2. Algebraic hulls. A group $\Gamma$ is polycyclic if it admits a sequence

$$
\Gamma=\Gamma_{0} \supset \Gamma_{1} \supset \cdots \supset \Gamma_{k}=\{e\}
$$

of subgroups such that each $\Gamma_{i}$ is normal in $\Gamma_{i-1}$ and $\Gamma_{i-1} / \Gamma_{i}$ is cyclic. For a polycyclic group $\Gamma$, we denote $\operatorname{rank} \Gamma=\sum_{i=1}^{i=k} \operatorname{rank} \Gamma_{i-1} / \Gamma_{i}$. Let $G$ be a simply connected solvable Lie group, and let $\Gamma$ to be a lattice in $G$. Then $\Gamma$ is torsion-free polycyclic and $\operatorname{dim} G=\operatorname{rank} \Gamma$ (see $[\mathbf{2 6}$, Proposition 3.7]). Let $\rho: G \rightarrow G L_{n}(\mathbb{C})$, for $g \in G$ be a representation. Let $\mathbf{G}$ and $\mathbf{G}^{\prime}$ be the Zariski-closures of $\rho(G)$ and $\rho(\Gamma)$ in $G L_{n}(\mathbb{C})$. Then we have $\mathbf{U}(\mathbf{G})=\mathbf{U}\left(\mathbf{G}^{\prime}\right)$ (see $[\mathbf{2 6}$, Theorem 3.2]).

We review the algebraic hulls. 
Proposition 2.1. ([26, Proposition 4.40]) Let $G$ be a simply connected solvable Lie group (resp. torsion-free polycyclic group). Then there exists a unique $\mathbb{R}$-algebraic group $\mathbf{H}_{G}$ with an injective group homomorphism $\psi: G \rightarrow \mathbf{H}_{G}(\mathbb{R})$ so that:

(1) $\psi(G)$ is Zariski-dense in $\mathbf{H}_{\mathbf{G}}$.

(2) $Z_{\mathbf{H}_{G}}\left(\mathbf{U}\left(\mathbf{H}_{G}\right)\right) \subset \mathbf{U}\left(\mathbf{H}_{G}\right)$.

(3) $\operatorname{dim} \mathbf{U}\left(\mathbf{H}_{G}\right)=\operatorname{dim} G($ resp. $\operatorname{rank} G)$.

Such $\mathbf{H}_{G}$ is called the algebraic hull of $G$.

We denote $\mathbf{U}_{G}=\mathbf{U}\left(\mathbf{H}_{G}\right)$ and call $\mathbf{U}_{G}$ the unipotent hull of $G$.

2.3. Direct constructions of algebraic hulls. Let $\mathfrak{g}$ be a solvable Lie algebra, and $\mathfrak{n}=\left\{X \in \mathfrak{g} \mid \operatorname{ad}_{X}\right.$ is nilpotent $\}$. $\mathfrak{n}$ is the maximal nilpotent ideal of $\mathfrak{g}$ and called the nilradical of $\mathfrak{g}$. Then we have $[\mathfrak{g}, \mathfrak{g}] \subset \mathfrak{n}$. Consider the adjoint representation ad $: \mathfrak{g} \rightarrow D(\mathfrak{g})$ and the representation $\operatorname{ad}_{s}:$ $\mathfrak{g} \rightarrow D(\mathfrak{g})$ as Construction 1.1.

Let $\overline{\mathfrak{g}}=\operatorname{Imad}_{s} \ltimes \mathfrak{g}$ and

$$
\overline{\mathfrak{n}}=\left\{X-\operatorname{ad}_{s X} \in \overline{\mathfrak{g}} \mid X \in \mathfrak{g}\right\} .
$$

Then we have $[\mathfrak{g}, \mathfrak{g}] \subset \mathfrak{n} \subset \overline{\mathfrak{n}}$, and $\overline{\mathfrak{n}}$ is the nilradical of $\overline{\mathfrak{g}}$ (see [12]). Hence we have $\overline{\mathfrak{g}}=\operatorname{Im~ad}_{s} \ltimes \overline{\mathfrak{n}}$.

Lemma 2.2. Suppose $\mathfrak{g}=\mathbb{R}^{k} \ltimes_{\phi} \mathfrak{n}$ such that $\phi$ is a semi-simple action and $\mathfrak{n}$ is nilpotent. Then $\overline{\mathfrak{n}}=\mathbb{R}^{k} \oplus \mathfrak{n}$.

Proof. By assumption, for $X+Y \in \mathbb{R}^{k} \ltimes_{\phi} \mathfrak{n}$, we have $\operatorname{ad}_{s X+Y}=\operatorname{ad}_{X}$. Hence we have

$$
\left[X_{1}+Y_{1}-\operatorname{ad}_{s X_{1}+Y_{1}}, X_{2}+Y_{2}-\operatorname{ad}_{s X_{2}+Y_{2}}\right]=\left[X_{2}, Y_{2}\right]
$$

for $X_{1}+Y_{1}, X_{2}+Y_{2} \in \mathbb{R}^{k} \ltimes_{\phi} \mathfrak{n}$. Hence the lemma follows. q.e.d.

Let $G$ be a simply connected solvable Lie group and $\mathfrak{g}$ be the Lie algebra of $G$. Let $N$ be the subgroup of $G$ which corresponds to the nilradical $\mathfrak{n}$ of $\mathfrak{g}$. We consider the exponential map exp $: \mathfrak{g} \rightarrow G$. In general exp is not a diffeomorphism. But we have the useful property of exp as following.

Lemma 2.3. ([10, Lemma 3.3]) Let $V$ be a subvector space (not necessarily Lie algebra) $V$ of $\mathfrak{g}$ so that $\mathfrak{g}=V \oplus \mathfrak{n}$ as the direct sum of vector spaces. We define the map $F: \mathfrak{g}=V \oplus \mathfrak{n} \rightarrow G$ as $F(A+X)=$ $\exp (A) \exp (X)$ for $A \in V, X \in \mathfrak{n}$. Then $F$ is a diffeomorphism and we have the commutative diagram

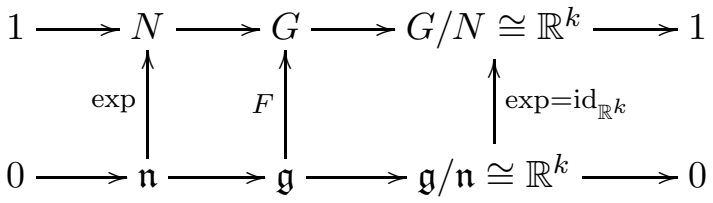

where $\operatorname{dim} G / N=k$. 
By this lemma, for $A \in V, X \in \mathfrak{n}$, the extension $\operatorname{Ad}_{s}: G \rightarrow \operatorname{Aut}\left(\mathfrak{g}_{\mathbb{C}}\right)$ is given by

$$
\operatorname{Ad}_{s}(\exp (A) \exp (X))=\exp \left(\left(\operatorname{ad}_{A}\right)_{s}\right)=\left(\exp \left(\operatorname{ad}_{A}\right)\right)_{s}
$$

and we have $\operatorname{Ad}_{s}(G)=\left\{\left(\exp \left(\operatorname{ad}_{A}\right)\right)_{s} \in \operatorname{Aut}\left(\mathfrak{g}_{\mathbb{C}}\right) \mid A \in V\right\}$. Let $\bar{G}=$ $\operatorname{Ad}_{s}(G) \ltimes G$. Then the Lie algebra of $\bar{G}$ is $\overline{\mathfrak{g}}$. For the nilradical $\bar{N}$ of $\bar{G}$, by the spritting $\overline{\mathfrak{g}}=\operatorname{Imad}_{s} \ltimes \overline{\mathfrak{n}}$ we have $\bar{G}=\operatorname{Ad}_{s}(G) \ltimes \bar{N}$ such that we can regard $\operatorname{Ad}_{s}(G) \subset \operatorname{Aut}(\overline{\mathrm{N}})$ and $\operatorname{Ad}_{s}(G)$ to consist of semi-simple automorphisms of $\bar{N}$. By the construction of $\overline{\mathfrak{n}}$ we have $\bar{G}=G \cdot \bar{N}$.

A simply connected nilpotent Lie group is considered to be the real points of a unipotent $\mathbb{R}$-algebraic group (see $[\mathbf{2 4}$, p. 43]) by the exponential map. We have the unipotent $\mathbb{R}$-algebraic group $\overline{\mathbf{N}}$ with $\overline{\mathbf{N}}(\mathbb{R})=\bar{N}$. We identify $\operatorname{Aut}_{\mathrm{a}}(\overline{\mathbf{N}})$ with $\operatorname{Aut}\left(\mathfrak{n}_{\mathbb{C}}\right)$, and $\operatorname{Aut}_{\mathrm{a}}(\overline{\mathbf{N}})$ has the $\mathbb{R}$-algebraic group structure with $\operatorname{Aut}_{a}(\overline{\mathbf{N}})(\mathbb{R})=\operatorname{Aut}(\bar{N})$. So we have the $\mathbb{R}$-algebraic

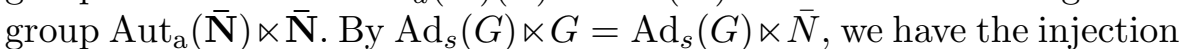
$I: G \rightarrow \operatorname{Aut}(\bar{N}) \ltimes \bar{N}=\operatorname{Aut}_{a}(\overline{\mathbf{N}}) \ltimes \overline{\mathbf{N}}(\mathbb{R})$. Let $\mathbf{G}$ be the Zariski-closure of $I(G)$ in $\operatorname{Aut}_{\mathrm{a}}(\overline{\mathbf{N}}) \ltimes \overline{\mathbf{N}}$.

Proposition 2.4. Let $\mathbf{T}$ be the Zariski-closure of $\operatorname{Ad}_{s}(G)$ in $\operatorname{Aut} \overline{\mathbf{N}}$. Then we have $\mathbf{G}=\mathbf{T} \ltimes \overline{\mathbf{N}}$, and $\mathbf{G}$ is the algebraic hull of $G$ with the unipotent hull $\mathbf{U}_{G}=\overline{\mathbf{N}}$. Hence the Lie algebra of the unipotent hull $\mathbf{U}_{G}$ of $G$ is

$$
\overline{\mathfrak{n}}_{\mathbb{C}}=\left\{X-\operatorname{ad}_{s X} \in \overline{\mathfrak{g}}_{\mathbb{C}} \mid X \in \mathfrak{g}_{\mathbb{C}}\right\} .
$$

Proof. The algebraic group $\mathbf{T} \ltimes \overline{\mathbf{N}}$ is the Zariski-closure of $\operatorname{Ad}_{s}(G) \ltimes \bar{N}$

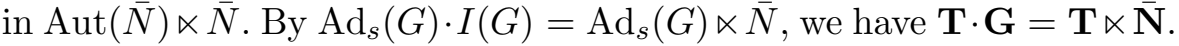
Since $\mathbf{T}$ is a diagonalizable algebraic group, we have $\overline{\mathbf{N}} \subset \mathbf{G}$. Otherwise, since $\mathbf{G} \subset \mathbf{T} \ltimes \overline{\mathbf{N}}$ is a connected solvable algebraic group, we have $\mathbf{U}(\mathbf{G})=\overline{\mathbf{N}} \cap \mathbf{G}=\overline{\mathbf{N}}$. Since we have $\operatorname{Ad}_{s}(G) \ltimes \bar{N}=G \cdot \bar{N}, \mathbf{G}$ is identified with the Zariski-closure of $\operatorname{Ad}_{s}(G) \ltimes \bar{N}$. Hence we have $\mathbf{G}=\mathbf{T} \ltimes \overline{\mathbf{N}}$. By $\operatorname{dim} G=\operatorname{dim} \bar{N}$, we can easily check that $\mathbf{T} \ltimes \overline{\mathbf{N}}$ is the algebraic hull of G.

q.e.d.

By this proposition, the Zariski-closure $\mathbf{T}$ of $\operatorname{Ad}_{s}(G)$ is a maximal torus of the algebraic hull of $G$. By the uniqueness of the algebraic hull (see [26, Lemma 4.41]), we have:

Lemma 2.5. Let $\mathbf{H}_{G}$ be the algebraic hull of $G$ and $q: \mathbf{H}_{G} \rightarrow$ $\mathbf{H}_{G} / \mathbf{U}_{G}$ the quotient map. Then for any injection $\psi: G \rightarrow \mathbf{H}_{G}(\mathbb{R})$ as in Proposition 2.1, there exists an isomorphism $\varphi: \mathbf{H}_{G} / \mathbf{U}_{G} \rightarrow \mathbf{T}$ such that the diagram

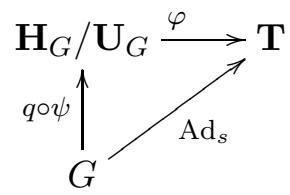

commutes. 
Lemma 2.6. Let $H_{G}=\mathbf{H}_{G}(\mathbb{R})$ be the real points of the algebraic hull of $G$. Let $\mathbf{T}$ be the Zariski-closure of $\operatorname{Ad}_{s}(G)$ in $\operatorname{Aut}\left(\mathfrak{g}_{\mathbb{C}}\right)$ and $T=\mathbf{T}(\mathbb{R})$ its real points. Then we have a semi-direct product

$$
H_{G}=T \ltimes G .
$$

Proof. By $\operatorname{Im}\left(\operatorname{ad}_{s}\right) \ltimes \overline{\mathfrak{n}}=\operatorname{Im}\left(\operatorname{ad}_{s}\right) \ltimes \mathfrak{g}$, we have $\operatorname{Ad}_{s}(G) \ltimes \bar{N}=\operatorname{Ad}_{s}(G) \ltimes$ $I(G)$. Hence the lemma follows from Proposition 2.4.

Proposition 2.7. ([19]) Let $G$ be a simply connected solvable Lie group. Then $\mathbf{U}_{G}$ is abelian if and only if $G=\mathbb{R}^{n} \ltimes_{\phi} \mathbb{R}^{m}$ such that the action $\phi: \mathbb{R}^{n} \rightarrow \operatorname{Aut}\left(\mathbb{R}^{m}\right)$ is semi-simple.

Proof. Suppose $\mathbf{U}_{G}$ is abelian. Then by Proposition 2.4, the Lie algebra $\overline{\mathfrak{n}}$ is abelian. By $\mathfrak{n} \subset \overline{\mathfrak{n}}$, the nilradical $\mathfrak{n}$ of $\mathfrak{g}$ is abelian. By $[\mathfrak{g}, \mathfrak{g}] \subset \mathfrak{n}$, $\mathfrak{g}$ is two-step solvable. We consider the lower central series $\mathfrak{g}^{i}$ as $\mathfrak{g}^{0}=\mathfrak{g}$ and $\mathfrak{g}^{i}=\left[\mathfrak{g}, \mathfrak{g}^{i-1}\right]$ for $i \geq 1$. We denote $\mathfrak{n}^{\prime}=\cap_{i=0}^{\infty} \mathfrak{g}^{i}$. Then by $[\mathbf{9}$, Lemma 4.1], we have $\mathfrak{g}=\mathfrak{g} / \mathfrak{n}^{\prime} \ltimes \mathfrak{n}^{\prime}$. For this decomposition, the subspace $\left\{X-\operatorname{ad}_{s X} \mid X \in \mathfrak{g} / \mathfrak{n}^{\prime}\right\} \subset \overline{\mathfrak{n}}$ is a Lie subalgebra of $\overline{\mathfrak{n}}$. Since $\mathfrak{g} / \mathfrak{n}^{\prime}$ is a nilpotent subalgebra of $\mathfrak{g}$, this space is identified with $\mathfrak{g} / \mathfrak{n}^{\prime}$. Thus since $\overline{\mathfrak{n}}$ is abelian, $\mathfrak{g} / \mathfrak{n}^{\prime}$ is also abelian. We show that the action of $\mathfrak{g} / \mathfrak{n}$ on $\mathfrak{n}$ is semisimple. Suppose for some $X \in \mathfrak{g} / \mathfrak{n}^{\prime}, \operatorname{ad}_{X}$ on $\mathfrak{n}$ is not semi-simple. Then $\operatorname{ad}_{X}-\operatorname{ad}_{s X}$ on $\mathfrak{n}$ is not trivial. Since we have $\overline{\mathfrak{n}}=\left\{X-\operatorname{ad}_{s X} \mid X \in \mathfrak{g}\right\}$, we have $[\overline{\mathfrak{n}}, \mathfrak{n}] \neq\{0\}$. This contradicts that $\overline{\mathfrak{n}}$ is abelian. Hence the action of $\mathfrak{g} / \mathfrak{n}$ on $\mathfrak{n}$ is semi-simple, and hence the first half of the proposition follows. The converse follows from Lemma 2.2.

q.e.d.

\section{Left-invariant forms and the cohomology of solvmanifolds}

Let $G$ be a simply connected solvable Lie group, $\mathfrak{g}$ the Lie algebra of $G$, and $\rho: G \rightarrow G L\left(V_{\rho}\right)$ a representation on a $\mathbb{C}$-vector space $V_{\rho}$. We consider the cochain complex $\bigwedge \mathfrak{g}^{*}$ with the derivation $d$ which is the dual to the Lie bracket of $\mathfrak{g}$. Then $\bigwedge \mathfrak{g}_{\mathbb{C}}^{*} \otimes V_{\rho}$ is a cochain complex with the derivation $d_{\rho}=d+\rho_{*}$, where $\rho_{*}$ is the derivation of $\rho$, and consider $\rho_{*} \in \mathfrak{g}_{\mathbb{C}}^{*} \otimes \mathfrak{g l}\left(V_{\rho}\right)$. We can consider the cochain complex $\left(\bigwedge \mathfrak{g}_{\mathbb{C}}^{*} \otimes V_{\rho}, d_{\rho}\right)$ to be the twisted $G$-invariant differential forms on $G$. Consider the cochain complex $A_{\mathbb{C}}^{*}(G) \otimes V_{\rho}$ with the derivation $d$ such that

$$
d(\omega \otimes v)=(d \omega) \otimes v \quad \omega \in A_{\mathbb{C}}^{*}(G), \quad v \in V_{\rho} .
$$

By the left action of $G$ (given by $(g \cdot f)(x)=f\left(g^{-1} x\right), f \in C^{\infty}(G), g \in$ $G$ ) and $\rho$, we have the action of $G$ on $A_{\mathbb{C}}^{*}(G) \otimes V_{\rho}$. Denote $\left(A_{\mathbb{C}}^{*}(G) \otimes V_{\rho}\right)^{G}$ the $G$-invariant elements of $A_{\mathbb{C}}^{*}(G) \otimes V_{\rho}$. Then we have an isomorphism

$$
\left(A_{\mathbb{C}}^{*}(G) \otimes V_{\rho}\right)^{G} \cong \bigwedge \mathfrak{g}_{\mathbb{C}}^{*} \otimes V_{\rho} .
$$

Suppose $G$ has a lattice $\Gamma$. Since $\pi_{1}(G / \Gamma)=\Gamma$, we have a flat vector bundle $E_{\rho_{\left.\right|_{\Gamma}}}$ with flat connection $D_{\rho_{\left.\right|_{\Gamma}}}$ on $G / \Gamma$ whose monodromy is $\rho_{\left.\right|_{\Gamma}}$. Let $A^{*}\left(G / \Gamma, E_{\rho_{\left.\right|_{\Gamma}}}\right)$ be the cochain complex of $E_{\rho_{\left.\right|_{\Gamma}}}$-valued differential 
forms with the derivation $D_{\rho_{\left.\right|_{\Gamma}}}$. Consider the cochain complex $A_{\mathbb{C}}^{*}(G) \otimes$ $V_{\rho}$ with derivation $d$ such that

$$
d(\omega \otimes v)=(d \omega) \otimes v \quad \omega \in A_{\mathbb{C}}^{*}(G), \quad v \in V_{\rho} .
$$

Then we have the $G$-action on $A_{\mathbb{C}}^{*}(G) \otimes V_{\rho}$ and denote $\left(A_{\mathbb{C}}^{*}(G) \otimes V_{\rho}\right)^{\Gamma}$ the subcomplex of $\Gamma$-invariant elements of $A_{\mathbb{C}}^{*}(G) \otimes V_{\rho}$. We have the isomorphism $\left(A_{\mathbb{C}}^{*}(G) \otimes V_{\rho}\right)^{\Gamma} \cong A^{*}\left(G / \Gamma, E_{\rho_{\mid \Gamma}}\right)$. Thus we have

$$
\bigwedge \mathfrak{g}_{\mathbb{C}}^{*} \otimes V_{\rho} \cong\left(A_{\mathbb{C}}^{*}(G) \otimes V_{\rho}\right)^{G} \subset\left(A_{\mathbb{C}}^{*}(G) \otimes V_{\rho}\right)^{\Gamma} \cong A^{*}\left(G / \Gamma, E_{\rho_{\left.\right|_{\Gamma}}}\right)
$$

and we have the inclusion $\bigwedge \mathfrak{g}_{\mathbb{C}}^{*} \otimes V_{\rho} \rightarrow A^{*}\left(G / \Gamma, E_{\rho_{\mid}}\right)$.

We call a representation $\rho \Gamma$-admissible if for the representation $\rho \oplus$ Ad $: G \rightarrow G L_{n}(\mathbb{C}) \times \operatorname{Aut}\left(\mathfrak{g}_{\mathbb{C}}\right),(\rho \oplus \operatorname{Ad})(G)$ and $(\rho \oplus \operatorname{Ad})(\Gamma)$ have the same Zariski-closure in $G L_{n}(\mathbb{C}) \times \operatorname{Aut}\left(\mathfrak{g}_{\mathbb{C}}\right)$.

Theorem 3.1. ([20], [26, Theorem 7.26]) If $\rho$ is $\Gamma$-admissible, then the inclusion

$$
\bigwedge \mathfrak{g}_{\mathbb{C}}^{*} \otimes V_{\rho} \rightarrow A^{*}\left(G / \Gamma, E_{\rho_{\mid \Gamma}}\right)
$$

induces a cohomology isomorphism.

Proposition 3.2. Let $G$ be a simply connected solvable Lie group with a lattice $\Gamma$. We suppose $\operatorname{Ad}(G)$ and $\operatorname{Ad}(\Gamma)$ have the same Zariskiclosure in $\operatorname{Aut}\left(\mathfrak{g}_{\mathbb{C}}\right)$. We consider the diagonalizable representation $\operatorname{Ad}_{s}$ : $G \rightarrow \operatorname{Aut}(G)$. Let $\mathbf{T}$ be the Zariski-closure of $\operatorname{Ad}_{s}(G)$ and $\alpha$ be a character of $\mathbf{T}$. Then $\alpha \circ \operatorname{Ad}_{s}$ is $\Gamma$-admissible.

Proof. Let $\mathbf{G}$ be the Zariski-closure of $\operatorname{Ad}(G)$ in $\operatorname{Aut}\left(\mathfrak{g}_{\mathbb{C}}\right)$. We first show that $\mathbf{T}$ is a maximal torus of $\mathbf{G}$. For the direct sum $\mathfrak{g}=V \oplus \mathfrak{n}$ as Construction 1.1, the map $F: V \oplus \mathfrak{n} \rightarrow G$ defined by $F(A+X)=$ $\exp (A) \exp (X)$ for $A \in V, X \in \mathfrak{n}$ is a diffeomorphism (see [10, Lemma 3.3]). For $A \in V$, we consider the Jordan decomposition $\operatorname{Ad}(\exp (A))=$ $\exp \left(\left(\operatorname{ad}_{A}\right)_{s}\right) \exp \left(\left(\operatorname{ad}_{A}\right)_{n}\right)$. Then we have $\exp \left(\left(\operatorname{ad}_{A}\right)_{s}\right), \exp \left(\left(\operatorname{ad}_{A}\right)_{n}\right) \in \mathbf{G}$. For $X \in \mathfrak{n}$, we have $\exp \left(\operatorname{ad}_{X}\right) \in \mathbf{U}(\mathbf{G})$. Hence we have $\operatorname{Ad}(G) \subset$ $\mathbf{T U}(\mathbf{G}) \subset \mathbf{G}$. Since $\mathbf{G}$ is a Zariski-closure of $\operatorname{Ad}(G), \mathbf{G}=\mathbf{T U}(\mathbf{G})$. Thus $\mathbf{T}$ is a maximal torus of $\mathbf{G}$.

We take a spritting $\mathbf{G}=\mathbf{T} \ltimes \mathbf{U}(\mathbf{G})$. We consider the algebraic group

$$
\mathbf{G}^{\prime}=\left\{(\alpha(t),(t, u)) \in \mathbb{C}^{*} \times \mathbf{G} \mid(t, u) \in \mathbf{T} \ltimes \mathbf{U}(\mathbf{G})\right\} .
$$

Then we have

$$
\begin{aligned}
& \left(\alpha \circ \operatorname{Ad}_{s} \oplus \operatorname{Ad}\right)(G) \\
& \quad=\left\{\left(\alpha\left(\exp \left(\left(\operatorname{ad}_{A}\right)_{s}\right)\right), \exp \left(\operatorname{ad}_{A}\right) \exp \left(\operatorname{ad}_{X}\right)\right) \mid A+X \in V \oplus \mathfrak{n}\right\} \\
& \subset \mathbf{G}^{\prime} .
\end{aligned}
$$

Since $\mathbf{G}$ is a Zariski-closure of $\operatorname{Ad}(G),\left(\alpha \circ \operatorname{Ad}_{s} \oplus \operatorname{Ad}\right)(G)$ is Zariskidense in $\mathbf{G}^{\prime}$. Since $\operatorname{Ad}(G)$ and $\operatorname{Ad}(\Gamma)$ have the same Zariski-closure, 
$\left(\alpha \circ \operatorname{Ad}_{s} \oplus \operatorname{Ad}\right)(G)$ and $\left(\alpha \circ \operatorname{Ad}_{s} \oplus \operatorname{Ad}\right)(\Gamma)$ have the same Zariski-closure $\mathbf{G}^{\prime}$.

q.e.d.

\section{Hain's DGAs}

4.1. Constructions. Let $M$ be a $C^{\infty}$-manifold, $\mathbf{S}$ be a reductive algebraic group, and $\rho: \pi_{1}(M, x) \rightarrow \mathbf{S}$ be a representation. We assume the image of $\rho$ is Zariski-dense in $\mathbf{S}$. Let $\left\{V_{\alpha}\right\}$ be the set of irreducible representations of $\mathbf{S},\left(E_{\alpha}, D_{\alpha}\right)$ a flat bundle with the monodromy $\alpha \circ \rho$, and $A^{*}\left(M, E_{\alpha}\right)$ the space of $E_{\rho}$-valued $C^{\infty}$-differential forms. Then we have an algebra isomorphism of $\bigoplus_{\alpha} V_{\alpha} \otimes V_{\alpha}^{*}$ and the coordinate ring $\mathbb{C}[\mathbf{S}]$ of $\mathbf{S}$ (see [14, Section 3]). Denote

$$
A^{*}\left(M, \mathcal{O}_{\rho}\right)=\bigoplus_{\alpha} A^{*}\left(M, E_{\alpha}\right) \otimes V_{\alpha}^{*}
$$

and $D=\bigoplus_{\alpha} D_{\alpha}$. Then by the wedge product, $\left(A\left(M, \mathcal{O}_{\rho}\right), D\right)$ is a cohomologically connected DGA with coefficients in $\mathbb{C}$.

Suppose $\mathbf{S}$ is a diagonal algebraic group. Then $\left\{V_{\alpha}\right\}$ is the set of one-dimensional representations for all algebraic characters $\alpha$ of $\mathbf{T}$, and $\left(E_{\alpha}, D_{\alpha}\right)$ are rank one flat bundles with the monodromy $\alpha \circ \rho$. In this case, for characters $\alpha$ and $\beta$, we have the wedge product $A^{*}\left(M, E_{\alpha}\right) \otimes$ $A^{*}\left(M, E_{\beta}\right) \rightarrow A^{*}\left(M, E_{\alpha \beta}\right)$ and $D_{\alpha \beta}\left(\psi_{\alpha} \wedge \psi_{\beta}\right)=D_{\alpha} \psi_{\alpha} \wedge \psi_{\beta}+(-1)^{p} \psi_{\alpha} \wedge$ $D_{\beta} \psi_{\beta}$ for $\psi_{\alpha} \in A^{p}\left(M, E_{\alpha}\right), \psi_{\beta} \in A^{q}\left(M, E_{\beta}\right)$ (see [22] for details in this case).

4.2. Formality and the hard Lefschetz properties of compact Kähler manifolds. In this subsection we will prove the following theorem by theories of Higgs bundles studied by Simpson.

Theorem 4.1. Let $M$ be a compact Kähler manifold with a Kähler form $\omega$ and $\rho: \pi_{1}(M) \rightarrow \mathbf{S}$ a representation to a reductive algebraic group $\mathbf{S}$ with the Zariski-dense image. Then the following conditions hold:

(A) (formality) The $D G A A^{*}\left(M, \mathcal{O}_{\rho}\right)$ is formal.

(B) (hard Lefschetz) For any $0 \leq i \leq n$, the linear operator

$$
[\omega]^{i} \wedge: H^{n-i}\left(A^{*}\left(M, \mathcal{O}_{\rho}\right)\right) \rightarrow H^{n+i}\left(A^{*}\left(M, \mathcal{O}_{\rho}\right)\right)
$$

is an isomorphism where $\operatorname{dim}_{\mathbb{R}} M=2 n$.

Let $M$ be a compact Kähler manifold and $E$ a holomorphic vector bundle on $M$ with the Dolbeault operator $\bar{\partial}$. For an $\operatorname{End}(E)$-valued holomorphic form $\theta$, we denote $D^{\prime \prime}=\bar{\partial}+\theta$. We call $\left(E, D^{\prime \prime}\right)$ a Higgs bundle if it satisfies the Leibniz rule: $D^{\prime \prime}(a e)=\bar{\partial}(a) e+(-1)^{p} D^{\prime \prime}(e)$ for $a \in A^{p}(M), e \in A^{0}(E)$ and the integrability $\left(D^{\prime \prime}\right)^{2}=0$. Let $h$ be a Hermitian metric on $E$. For a Higgs bundle $\left(E, D^{\prime \prime}=\bar{\partial}+\theta\right)$, we define $D_{h}^{\prime}=\partial_{h}+\bar{\theta}_{h}$ as follows: $\partial_{h}$ is the unique operator which satisfies

$$
h(\bar{\partial} e, f)+h\left(e, \partial_{h} f\right)=\bar{\partial} h(e, f)
$$


and $\bar{\theta}_{h}$ is defined by $(\theta e, f)=\left(e, \bar{\theta}_{h} f\right)$. Let $D_{h}=D_{h}^{\prime}+D^{\prime \prime}$. Then $D_{h}$ is a connection. We call a Higgs bundle $\left(E, D^{\prime \prime}, h\right)$ with a metric harmonic if $D_{h}$ is flat, i.e. $\left(D_{h}\right)^{2}=0$.

For two Higgs bundles $\left(E, D^{\prime \prime}\right),\left(F, D^{\prime \prime}\right)$ with metric $h_{E}, h_{F}$, the tensor product $\left(E \otimes F, D^{\prime \prime} \otimes 1+1 \otimes D^{\prime \prime}\right)$ is also a Higgs bundle, and $h_{E} \otimes h_{F}$ gives the connection $D_{h_{E} \otimes h_{F}}=D_{h_{E}} \otimes 1+1 \otimes D_{h_{F}}$ on $E \otimes F$. If $\left(E, D^{\prime \prime}, h_{E}\right)$ and $\left(F, D^{\prime \prime}, h_{F}\right)$ are harmonic, $\left(E \otimes F, D^{\prime \prime} \otimes 1+1 \otimes D^{\prime \prime}\right)$ is also a harmonic Higgs bundle with the flat connection $D_{h_{E}} \otimes 1+1 \otimes D_{h_{F}}$.

Theorem 4.2. ([28, Theorem 1]) Let $(E, D)$ be a flat bundle on $M$ whose monodromy is semi-simple. Then $D$ is given by a harmonic Higgs bundle $\left(E, D^{\prime \prime}, h\right)$, that is, $D=D_{h}$.

Theorem 4.3. ([28, Lemma 2.2]) Let $\left(E, D^{\prime \prime}, h\right)$ be a harmonic Higgs bundle with the flat connection $D=D^{\prime}+D^{\prime \prime}$. Then the inclusion

$$
\left(\operatorname{Ker} D^{\prime}, D^{\prime \prime}\right) \rightarrow\left(A^{*}(E), D\right)
$$

and the quotient

$$
\left(\operatorname{Ker} D^{\prime}, D^{\prime \prime}\right) \rightarrow\left(H_{D^{\prime}}\left(A^{*}(E)\right), D^{\prime \prime}\right)=\left(H_{D}^{*}\left(A^{*}(E)\right), 0\right)
$$

induce the cohomology isomorphisms.

Theorem 4.4. ([28, Lemma 2.6]) Let $\left(E, D^{\prime \prime}, h\right)$ be a harmonic Higgs bundle with the flat connection $D=D^{\prime}+D^{\prime \prime}$. Then for any $0 \leq i \leq n$ the linear operator

$$
[\omega]^{n-i} \wedge: H_{D}^{i}\left(A^{*}\left(E_{\rho}\right)\right) \rightarrow H_{D}^{2 n-i}\left(A^{*}\left(E_{\rho}\right)\right)
$$

is an isomorphism.

Proof of Theorem 4.1. By Theorems 4.2 and 4.4, the condition (B) holds. By Theorem 4.2 , for $\left(A^{*}\left(E_{\alpha}\right), D_{\alpha}\right)$, we have $D_{\alpha}=D_{\alpha}^{\prime}+D_{\alpha}^{\prime \prime}$ such that $D_{\alpha}^{\prime \prime}$ is a harmonic Higgs bundle. Denote $D^{\prime}=\bigoplus_{\alpha} D_{\alpha}^{\prime}$ and $D^{\prime \prime}=\bigoplus_{\alpha} D_{\alpha}^{\prime \prime}$. Then by properties of the Higgs bundle, $\left(\operatorname{Ker} D^{\prime}, D^{\prime \prime}\right)$ is a DGA, and the maps

$$
\left.\left(\operatorname{Ker} D^{\prime}, D^{\prime \prime}\right) \rightarrow\left(A^{*}\left(M, \mathcal{O}_{\rho}\right)\right), D\right)
$$

and

$$
\left(\operatorname{Ker} D^{\prime}, D^{\prime \prime}\right) \rightarrow\left(H_{D}^{*}\left(A^{*}\left(M, \mathcal{O}_{\rho}\right)\right), 0\right)
$$

are DGA homomorphisms, and thus quasi-isomorphisms by Theorem 4.3. Hence the condition (A) holds.

q.e.d.

\section{Minimal models of invariant forms on solvable Lie groups with local systems}

Let $G$ be a simply connected solvable Lie group and $\mathfrak{g}$ the Lie algebra of $G$. Consider the diagonal representation $\operatorname{Ad}_{s}$ as in Section 1 and the derivation $\operatorname{ad}_{s}$ of $\operatorname{Ad}_{s}$. For some basis $\left\{X_{1}, \ldots, X_{n}\right\}$ of $\mathfrak{g}_{\mathbb{C}}, \operatorname{Ad}_{s}$ is represented by diagonal matrices. Let $\mathbf{T}$ be the Zariski-closure of $\operatorname{Ad}_{s}(G)$ 
in $\operatorname{Aut}\left(\mathfrak{g}_{\mathbb{C}}\right)$. Let $\left\{V_{\alpha}\right\}$ be the set of one-dimensional representations for all characters $\alpha$ of $\mathbf{T}$. We consider $V_{\alpha}$ the representation of $\mathfrak{g}$ which is the derivation of $\alpha \circ \mathrm{Ad}_{s}$. Then we have the cochain complex of Lie algebra $\left(\bigwedge \mathfrak{g}_{\mathbb{C}}^{*} \otimes V_{\alpha}, d_{\alpha}\right)$. Denote $d=\bigoplus_{\alpha} d_{\alpha}$. Then $\left(\bigoplus_{\alpha} \bigwedge \mathfrak{g}_{\mathbb{C}}^{*} \otimes V_{\alpha}, d\right)$ is a cohomologically connected DGA with coefficients in $\mathbb{C}$ as the last section. By $\operatorname{Ad}_{s}(G) \subset \operatorname{Aut}\left(\mathfrak{g}_{\mathbb{C}}\right)$ we have $\mathbf{T} \subset \operatorname{Aut}\left(\mathfrak{g}_{\mathbb{C}}\right)$, and hence we have the action of $\mathbf{T}$ on $\bigoplus_{\alpha} \wedge \mathfrak{g}_{\mathbb{C}}^{*} \otimes V_{\alpha}$. Denote $\left(\bigoplus_{\alpha} \wedge \mathfrak{g}_{\mathbb{C}}^{*} \otimes V_{\alpha}\right)^{\mathbf{T}}$ the sub-DGA of $\bigoplus_{\alpha} \wedge \mathfrak{g}_{\mathbb{C}}^{*} \otimes V_{\alpha}$ which consists of the $\mathbf{T}$-invariant elements of $\bigoplus_{\alpha} \wedge \mathfrak{g}_{\mathbb{C}}^{*} \otimes V_{\alpha}$.

Lemma 5.1. We have an isomorphism

$$
H^{*}\left(\left(\bigoplus_{\alpha} \bigwedge \mathfrak{g}_{\mathbb{C}}^{*} \otimes V_{\alpha}\right)^{\mathbf{T}}\right) \cong H^{*}\left(\bigoplus_{\alpha} \bigwedge \mathfrak{g}_{\mathbb{C}}^{*} \otimes V_{\alpha}\right)
$$

Proof. We show that the action of $\operatorname{Ad}_{s}(G) \subset \mathbf{T}$ on the cohomology $H^{*}\left(\bigwedge \mathfrak{g}_{\mathbb{C}}^{*} \otimes V_{\alpha}\right)$ is trivial. Consider the direct sum $\mathfrak{g}=V \oplus \mathfrak{n}$ as Construction 1.1. Then we have $\operatorname{Ad}_{s}(G)=\operatorname{Ad}_{s}(\exp (V))$ by Lemma 2.3. For $A \in V$, the action $\operatorname{Ad}_{s}(\exp (A))$ on the cochain complex $\bigwedge \mathfrak{g}_{\mathbb{C}}^{*} \otimes V_{\alpha}$ is a semi-simple part of the action of $\exp (A)$ on $\bigwedge \mathfrak{g}_{\mathbb{C}}^{*} \otimes V_{\alpha}$ via $\operatorname{Ad} \otimes \alpha \circ \operatorname{Ad}_{s}$. Since the action of $G$ on the cohomology $H^{*}\left(\bigwedge \mathfrak{g}_{\mathbb{C}}^{*} \otimes V_{\alpha}\right)$ via $\operatorname{Ad} \otimes \alpha \circ \operatorname{Ad}_{s}$ is the extension of the Lie derivation on $H^{*}\left(\bigwedge \mathfrak{g}_{\mathbb{C}}^{*} \otimes V_{\alpha}\right)$, this $G$-action on $H^{*}\left(\bigwedge \mathfrak{g}_{\mathbb{C}}^{*} \otimes V_{\alpha}\right)$ is trivial. Hence for $A \in V$ the action of $\operatorname{Ad}_{s}(\exp (A))=$ $\left(\exp \left(\operatorname{ad}_{A}\right)\right)_{s}$ on the cohomology $H^{*}\left(\bigwedge \mathfrak{g}_{\mathbb{C}}^{*} \otimes V_{\alpha}\right)$ is trivial.

Since $\mathbf{T}$ is the Zariski-closure of $\operatorname{Ad}_{s}(G)$ in $\operatorname{Aut}\left(\mathfrak{g}_{\mathbb{C}}\right)$ and the action of $\mathbf{T}$ on $\bigwedge \mathfrak{g}_{\mathbb{C}}^{*} \otimes V_{\alpha}$ is algebraic, the action of $\mathbf{T}$ on $H^{*}\left(\bigwedge \mathfrak{g}_{\mathbb{C}}^{*} \otimes V_{\alpha}\right)$ is also trivial. Since the action of $\mathbf{T}$ on $\bigwedge \mathfrak{g}_{\mathbb{C}}^{*} \otimes V_{\alpha}$ is diagonalizable, we have an isomorphism

$$
H^{*}\left(\bigwedge \mathfrak{g}_{\mathbb{C}}^{*} \otimes V_{\alpha}\right) \cong H^{*}\left(\bigwedge \mathfrak{g}_{\mathbb{C}}^{*} \otimes V_{\alpha}\right)^{\mathbf{T}} \cong H^{*}\left(\left(\bigwedge \mathfrak{g}_{\mathbb{C}}^{*} \otimes V_{\alpha}\right)^{\mathbf{T}}\right) .
$$

Hence we have the lemma.

q.e.d.

Consider the unipotent hull $\mathbf{U}_{G}$ of $G$. Let $\mathfrak{u}$ be the $\mathbb{C}$-Lie algebra of $\mathbf{U}_{G}$ and $\mathfrak{u}^{*}$ the $\mathbb{C}$-dual space. We consider the DGA $\wedge \mathfrak{u}^{*}$ with coefficients in $\mathbb{C}$.

Lemma 5.2. We have an isomorphism of $D G A$

$$
\bigwedge \mathfrak{u}^{*} \cong\left(\bigoplus_{\alpha} \bigwedge \mathfrak{g}_{\mathbb{C}}^{*} \otimes V_{\alpha}\right)^{\mathbf{T}}
$$

Proof. Let $\left\{x_{1}, \ldots, x_{n}\right\}$ be the dual of a basis $\left\{X_{1}, \ldots, X_{n}\right\}$ of $\mathfrak{g}$ such that $\operatorname{Ad}_{s}$ is represented by diagonal matrices. We define characters $\alpha_{i}$ as $t \cdot X_{i}=\alpha_{i}(t) X_{i}$ for $t \in \mathbf{T}$. Then we have $t \cdot x_{i}=\alpha_{i}^{-1}(t) x_{i}$. Hence the vector space $\left(\bigoplus_{\alpha} \bigwedge^{1} \mathfrak{g}_{\mathbb{C}}^{*} \otimes V_{\alpha}\right)^{\mathbf{T}}$ is spanned by $\left\{x_{1} \otimes v_{\alpha_{1}}, \ldots, x_{n} \otimes v_{\alpha_{n}}\right\}$ where $V_{\alpha_{i}} \ni v_{\alpha_{i}} \neq 0$. For

$$
\omega=\sum_{i_{1}, \ldots, i_{p}, \alpha} a_{i_{1}, \ldots, i_{p}, \alpha} x_{i_{1}} \wedge \cdots \wedge x_{i_{p}} v_{\alpha} \in\left(\bigoplus_{\alpha} \bigwedge^{p} \mathfrak{g}_{\mathbb{C}}^{*} \otimes V_{\alpha}\right)^{\mathbf{T}},
$$


since any $x_{i_{1}} \wedge \cdots \wedge x_{i_{p}} v_{\alpha}$ is an eigenvector of the action of $\mathbf{T}$, if $a_{i_{1}, \ldots, i_{p}, \alpha} \neq 0$ then $x_{i_{1}} \wedge \cdots \wedge x_{i_{p}} v_{\alpha}$ is also a $\mathbf{T}$-invariant element. Since we have

$$
t \cdot x_{i_{1}} \wedge \cdots \wedge x_{i_{p}}=\alpha_{i_{1}}^{-1}(t) \cdots \alpha_{i_{p}}^{-1}(t) x_{i_{1}} \wedge \cdots \wedge x_{i_{p}}
$$

for $t \in \mathbf{T}$, we have

$$
x_{i_{1}} \wedge \cdots \wedge x_{i_{p}} \otimes v_{\alpha}=x_{i_{1}} v_{\alpha_{i_{1}}} \wedge \cdots \wedge x_{i_{p}} v_{\alpha_{i_{p}}} .
$$

Thus the DGA $\left(\bigoplus_{\alpha} \wedge \mathfrak{g}_{\mathbb{C}}^{*} \otimes V_{\alpha}\right)^{\mathbf{T}}$ is generated by $\left\{x_{1} \otimes v_{\alpha_{1}}, \ldots, x_{n} \otimes v_{\alpha_{n}}\right\}$. Consider the Maurer-Cartan equations

$$
d x_{k}=-\sum_{i j} c_{i j}^{k} x_{i} \wedge x_{j}
$$

and denote $\operatorname{ad}_{s X_{i}}\left(X_{j}\right)=a_{i j} X_{j}$. Since $\operatorname{Ad}_{s g}\left(X_{k}\right)=\alpha_{i}\left(\operatorname{Ad}_{s g}\right) X_{k}$ for $g \in$ $G$, we have $d v_{\alpha_{k}}=\sum_{i=1}^{n} \operatorname{ad}_{s X_{i}}\left(X_{k}\right) x_{i} v_{\alpha_{k}}=\sum_{i=1}^{n} a_{i k} x_{i} v_{\alpha_{k}}$. Then we have

$$
d_{\alpha_{k}}\left(x_{k} \otimes v_{\alpha_{k}}\right)=-\sum_{i j}\left(c_{i j}^{k} x_{i} \wedge x_{j} \otimes v_{\alpha_{k}}-a_{i k} x_{i} \wedge x_{k} \otimes v_{\alpha_{k}}\right) .
$$

Hence the DGA $\left(\bigoplus_{\alpha} \wedge \mathfrak{g}_{\mathbb{C}}^{*} \otimes V_{\alpha}\right)^{\mathbf{T}}$ is isomorphic to a free DGA generated by degree 1 elements $\left\{y_{1}, \ldots, y_{n}\right\}$ such that

$$
d\left(y_{k}\right)=-\sum_{i j}\left(c_{i j}^{k} y_{i} \wedge y_{j}-a_{i k} y_{i} \wedge y_{k}\right)
$$

Let $\mathfrak{h}$ be the Lie algebra which is the dual of the free DGA $\left(\bigoplus_{\alpha} \wedge \mathfrak{g}_{\mathbb{C}}^{*} \otimes\right.$ $\left.V_{\alpha}\right)^{\mathbf{T}}$ and $\left\{Y_{1}, \ldots, Y_{n}\right\}$ the dual basis of $\left\{y_{1}, \ldots, y_{n}\right\}$. It is sufficient to show $\mathfrak{h} \cong \mathfrak{u}$. Then the bracket of $\mathfrak{h}$ is given by

$$
\left[Y_{i}, Y_{j}\right]=\sum_{k} c_{i j}^{k} Y_{k}-a_{i j} Y_{j}+a_{j i} Y_{i}
$$

Otherwise, by Section 2.3, we have $\mathfrak{u} \cong\left\{X-\operatorname{ad}_{s X} \mid X \in \mathfrak{g}_{\mathbb{C}}\right\} \subset D\left(\mathfrak{g}_{\mathbb{C}}\right) \ltimes$ $\mathfrak{g}_{\mathbb{C}}$. For the basis $\left\{X_{1}-\operatorname{ad}_{s X_{1}}, \ldots, X_{n}-\operatorname{ad}_{s X_{n}}\right\}$ of $\mathfrak{u}$, we have

$$
\left[X_{i}-\operatorname{ad}_{s X_{i}}, X_{j}-\operatorname{ad}_{s X_{j}}\right]=\sum_{k} c_{i j}^{k} X_{k}-a_{i j} X_{j}+a_{j i} X_{i}
$$

By $[\mathfrak{g}, \mathfrak{g}] \subset \mathfrak{n}$, we have $[\mathfrak{u}, \mathfrak{u}] \subset \mathfrak{n}_{\mathbb{C}}$, where $\mathfrak{n}$ is the nilradical of $\mathfrak{g}$. By this we have

$$
\sum_{k} c_{i j}^{k} X_{k}-a_{i j} X_{j}+a_{j i} X_{i} \in \mathfrak{n}_{\mathbb{C}}
$$

and hence we have

$$
a d_{s \sum_{k} c_{i j}^{k} X_{k}-a_{i j} X_{j}+a_{j i} X_{i}}=0 .
$$


This gives

$$
\begin{aligned}
& {\left[X_{i}-\operatorname{ad}_{s X_{i}}, X_{j}-\operatorname{ad}_{s X_{j}}\right]} \\
& \quad=\sum_{k} c_{i j}^{k}\left(X_{k}-\operatorname{ad}_{s X_{k}}\right)-a_{i j}\left(X_{j}-\operatorname{ad}_{s X_{j}}\right)+a_{j i}\left(X_{i}-\operatorname{ad}_{s X_{i}}\right) .
\end{aligned}
$$

This gives an isomorphism $\mathfrak{h} \cong \mathfrak{u}$. Hence the lemma follows. $\quad$ q.e.d.

Since $\operatorname{Ad}_{s}(G)$ is Zariski-dense in $\mathbf{T}, \operatorname{Ad}_{s}(G)$-invariant elements are also $\mathbf{T}$-invariant. In particular, we have the following lemma.

Lemma 5.3. Let $T=\mathbf{T}(\mathbb{R})$ be the real points of $\mathbf{T}$. Then we have

$$
\left(\bigoplus_{\alpha} \bigwedge \mathfrak{g}_{\mathbb{C}}^{*} \otimes V_{\alpha}\right)^{T} \cong\left(\bigoplus_{\alpha} \bigwedge \mathfrak{g}_{\mathbb{C}}^{*} \otimes V_{\alpha}\right)^{\mathbf{T}} \cong \bigwedge \mathfrak{u}^{*}
$$

Later we use this lemma.

Denote $A^{*}\left(\mathfrak{g}_{\mathbb{C}}, \operatorname{ad}_{s}\right)=\bigoplus_{\alpha} \wedge \mathfrak{g}_{\mathbb{C}}^{*} \otimes V_{\alpha}$. By Lemmas 5.1 and 5.2 we have:

Theorem 5.4. We have a quasi-isomorphism of DGAs

$$
\bigwedge \mathfrak{u}^{*} \rightarrow A^{*}\left(\mathfrak{g}_{\mathbb{C}}, \operatorname{ad}_{s}\right) .
$$

Thus $\bigwedge \mathfrak{u}^{*}$ is the minimal model of $A^{*}\left(\mathfrak{g}_{\mathbb{C}}, \operatorname{ad}_{s}\right)$.

\section{Cohomology of $A^{*}\left(G / \Gamma, \mathcal{O}_{\left.\mathrm{Ad}_{s \mid}\right|_{\Gamma}}\right)$}

Consider the two DGAs $A^{*}\left(\mathfrak{g}_{\mathbb{C}}, \operatorname{ad}_{s}\right)$ and $A^{*}\left(G / \Gamma, \mathcal{O}_{\operatorname{Ad}_{\left.s\right|_{\Gamma}}}\right)$. For any character $\alpha$ of an algebraic group $\mathbf{T}$ which is the Zariski-closure of $\operatorname{Ad}_{s}(G)$ in $\operatorname{Aut}\left(\mathfrak{g}_{\mathbb{C}}\right)$, we have the inclusion

$$
\bigwedge \mathfrak{g}_{\mathbb{C}}^{*} \otimes V_{\alpha} \cong\left(A_{\mathbb{C}}^{*}(G) \otimes V_{\alpha}\right)^{G} \subset\left(A_{\mathbb{C}}^{*}(G) \otimes V_{\alpha}\right)^{\Gamma} \cong A^{*}\left(G / \Gamma, E_{\left.\alpha \circ A_{s}\right|_{\Gamma}}\right) .
$$

Thus we have the morphism of DGAs

$$
\phi: A^{*}\left(\mathfrak{g}_{\mathbb{C}}, \operatorname{ad}_{s}\right) \rightarrow A^{*}\left(G / \Gamma, \mathcal{O}_{\operatorname{Ad}_{s \mid \Gamma}}\right) .
$$

Proposition 6.1. The morphism $\phi: A^{*}\left(\mathfrak{g}_{\mathbb{C}}, \operatorname{ad}_{s}\right) \rightarrow A^{*}\left(G / \Gamma, \mathcal{O}_{\operatorname{Ad}_{\left.s\right|_{\Gamma}}}\right)$ is injective and the induced map

$$
\phi^{*}: H^{*}\left(A^{*}\left(\mathfrak{g}_{\mathbb{C}}, \operatorname{ad}_{s}\right)\right) \rightarrow H^{*}\left(A^{*}\left(G / \Gamma, \mathcal{O}_{\operatorname{Ad}_{\left.s\right|_{\Gamma}}}\right)\right)
$$

is also injective.

Proof. Since $G$ has a lattice $\Gamma, G$ is unimodular (see [26, Remark 1.9]). Choose a Haar measure $d \mu$ such that the volume of $G / \Gamma$ is 1 . We define a map $\varphi_{\alpha}:\left(A_{\mathbb{C}}^{*}(G) \otimes V_{\alpha}\right)^{\Gamma} \rightarrow \bigwedge \mathfrak{g}_{\mathbb{C}}^{*} \otimes V_{\alpha}$ as

$$
\varphi_{\alpha}\left(\omega \otimes v_{\alpha}\right)\left(X_{1}, \ldots, X_{p}\right)=\int_{G / \Gamma} \frac{\omega_{x}}{\alpha(x)}\left(X_{1}, \ldots, X_{p}\right) d \mu \cdot v_{\alpha}
$$


for $\omega \otimes v_{\alpha} \in\left(A_{\mathbb{C}}^{p}(G) \otimes V_{\alpha}\right)^{\Gamma}, X_{1}, \ldots, X_{p} \in \mathfrak{g}_{C}$. Then each $\varphi_{\alpha}$ is a morphism of cochain complexes and we have $\varphi_{\alpha} \circ \phi_{\left.\right|_{\wedge \mathfrak{g}_{\mathbb{C}}^{*} \otimes V_{\alpha}}}=\operatorname{id}_{\left.\right|_{\wedge \mathfrak{g}_{\mathbb{C}}^{*} \otimes V_{\alpha}}}$ (see [26, Remark 7.30]). Thus the restriction

$$
\phi^{*}: H^{*}\left(\bigwedge \mathfrak{g}_{\mathbb{C}}^{*} \otimes V_{\alpha}\right) \rightarrow H^{*}\left(A^{*}\left(G / \Gamma, E_{\alpha}\right)\right)
$$

is injective. By this it is sufficient to show that two distinct characters $\alpha, \beta$ with $\alpha \circ \operatorname{Ad}_{\left.s\right|_{\Gamma}}=\beta \circ \operatorname{Ad}_{\left.s\right|_{\Gamma}}$ satisfy $\varphi_{\beta} \circ \phi_{\left.\right|_{\Lambda \mathfrak{g}_{\mathbb{C}}^{*} \otimes V_{\alpha}}}=0$. For $\omega \otimes v_{\alpha} \in$ $\bigwedge \mathfrak{g}_{\mathbb{C}}^{*} \otimes V_{\alpha}$, we have

$$
\varphi_{\beta} \circ \phi_{\left.\right|_{\wedge \mathfrak{g}_{\mathbb{C}}^{*} \otimes V_{\alpha}}}\left(\omega \otimes v_{\alpha}\right)=\int_{G / \Gamma} \frac{\alpha(x)}{\beta(x)} \omega_{x}\left(X_{1}, \ldots, X_{p}\right) d \mu \cdot v_{\alpha} .
$$

Since $\omega \in \bigwedge \mathfrak{g}_{\mathbb{C}}^{*}, \omega_{x}\left(X_{1}, \ldots, X_{p}\right)$ is constant on $G / \Gamma$. Let $\lambda=\frac{\beta}{\alpha} d\left(\frac{\alpha}{\beta}\right)$. Then $\lambda$ is a $G$-invariant form. Choose $\eta \in \wedge \mathfrak{g}_{\mathbb{C}}^{*}$ such that $\lambda \wedge \eta=d \mu$. Then we have

$$
d\left(\frac{\alpha}{\beta} \eta\right)=\frac{\alpha}{\beta} \lambda \wedge \eta=\frac{\alpha}{\beta} d \mu .
$$

By $\alpha \circ \operatorname{Ad}_{\left.s\right|_{\Gamma}}=\beta \circ \operatorname{Ad}_{\left.s\right|_{\Gamma}}, \frac{\alpha}{\beta} \eta$ is $\Gamma$-invariant and we can consider $\frac{\alpha}{\beta} \eta$ a differential form on $G / \Gamma$. Hence by Stokes' theorem, we have

$$
\begin{aligned}
\int_{G / \Gamma} \frac{\alpha(x)}{\beta(x)} \omega_{x}\left(X_{1}, \ldots, X_{p}\right) d \mu= & \omega\left(X_{1}, \ldots, X_{p}\right) \int_{G / \Gamma} \frac{\alpha(x)}{\beta(x)} d \mu \\
& =\omega\left(X_{1}, \ldots, X_{p}\right) \int_{G / \Gamma} d\left(\frac{\alpha}{\beta} \eta\right)=0 .
\end{aligned}
$$

This proves the proposition.

q.e.d.

Corollary 6.2. Let $G$ be a simply connected solvable Lie group with a lattice $\Gamma$. We suppose $\operatorname{Ad}(G)$ and $\operatorname{Ad}(\Gamma)$ have the same Zariski-closure in $\operatorname{Aut}\left(\mathfrak{g}_{\mathbb{C}}\right)$. Then we have an isomorphism

$$
H^{*}\left(A^{*}\left(G / \Gamma, \mathcal{O}_{\operatorname{Ad}_{s \mid \Gamma}}\right)\right) \cong H^{*}\left(A^{*}\left(\mathfrak{g}_{\mathbb{C}}, \operatorname{ad}_{s}\right)\right) .
$$

Proof. Let $\mathbf{T}$ be the Zariski-closure of $\operatorname{Ad}_{s}(G)$. For any 1-dimensional representation $V_{\alpha}$ of $\mathbf{T}$ given by a character $\alpha$ of $\mathbf{T}$, we consider a flat bundle $E_{\alpha}$ on $G / \Gamma$ given by the representation $\alpha \circ \operatorname{Ad}_{s}$ and the two cochain complex $A^{*}\left(G / \Gamma, E_{\alpha}\right)$ and $\bigwedge \mathfrak{g}_{\mathbb{C}}^{*} \otimes V_{\alpha}$ as above. Then since $\alpha \circ \operatorname{Ad}_{s}$ is $\Gamma$-admissible, by Theorem 3.1 we have an isomorphism

$$
H^{*}\left(\bigwedge \mathfrak{g}^{*} \otimes V_{\alpha}\right) \cong H^{*}\left(A^{*}\left(G / \Gamma, E_{\alpha}\right)\right) .
$$

By the definitions of $A^{*}\left(G / \Gamma, \mathcal{O}_{\mathrm{Ad}_{s_{\Gamma}}}\right)$ and $A^{*}\left(\mathfrak{g}_{\mathbb{C}}, \operatorname{ad}_{s}\right)$, the corollary follows. 


\section{Extensions}

In this section we extend Corollary 6.2 to the case of general solvmanifolds. To do this we consider infra-solvmanifolds which are generalizations of solvmanifolds.

7.1. Infra-solvmanifold. Let $G$ be a simply connected solvable Lie group. We consider the affine transformation group $\operatorname{Aut}(G) \ltimes G$ and the projection $p: \operatorname{Aut}(G) \ltimes G \rightarrow \operatorname{Aut}(G)$. Let $\Gamma \subset \operatorname{Aut}(G) \ltimes G$ be a discrete subgroup such that $p(\Gamma)$ is contained in a compact subgroup of $\operatorname{Aut}(G)$ and the quotient $G / \Gamma$ is compact. We call $G / \Gamma$ an infra-solvmanifold.

Theorem 7.1. [4, Theorem 1.5] For two infra-solvmanifolds $G_{1} / \Gamma_{1}$ and $G_{2} / \Gamma_{2}$, if $\Gamma_{1}$ is isomorphic to $\Gamma_{2}$, then $G_{1} / \Gamma_{1}$ is diffeomorphic to $G_{2} / \Gamma_{2}$.

7.2. Extensions for infra-solvmanifolds. Let $\Gamma$ be a torsion-free polycyclic group and $\mathbf{H}_{\Gamma}$ be the algebraic hull. Then there exists a finite index normal subgroup $\Delta$ of $\Gamma$ and a simply connected solvable subgroup $G$ of $\mathbf{H}_{\Gamma}$ such that $\Delta$ is a lattice of $G$, and $G$ and $\Delta$ have the same Zariski-closure in $\mathbf{H}_{\Gamma}$ (see [4, Proposition 2.9]). Since the Zariskiclosure of $\Delta$ in $\mathbf{H}_{\Gamma}$ is finite index normal subgroup of $\mathbf{H}_{\Gamma}$, this group is the algebraic hull $\mathbf{H}_{\Delta}$ of $\Delta$ by the properties in Proposition 2.1. By $\operatorname{rank} \Gamma=\operatorname{dim} G, \mathbf{H}_{\Delta}$ is also the algebraic hull $\mathbf{H}_{G}$ of $G$. Hence we have the commutative diagram

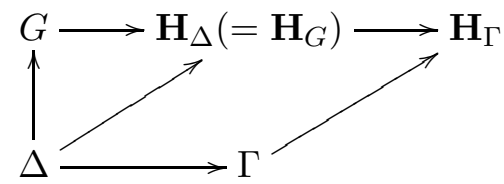

Since $\Delta$ is a finite index normal subgroup of $\Gamma$, by this diagram $\mathbf{H}_{\Delta}$ is a finite index normal subgroup of $\mathbf{H}_{\Gamma}$. We suppose $\mathbf{H}_{\Gamma} / \mathbf{U}_{\Gamma}$ is diagonalizable. Let $\mathbf{T}$ and $\mathbf{T}^{\prime}$ be maximal diagonalizable subgroups of $\mathbf{H}_{\Gamma}$ and $\mathbf{H}_{\Delta}$. Then we have decompositions $\mathbf{H}_{\Gamma}=\mathbf{T} \ltimes \mathbf{U}_{\Gamma}, \mathbf{H}_{\Delta}=\mathbf{T}^{\prime} \ltimes \mathbf{U}_{\Gamma}$. Since $\mathbf{T} / \mathbf{T}^{\prime}=\mathbf{H}_{\Gamma} / \mathbf{H}_{\Delta}$ is a finite group, we have a finite subgroup $\mathbf{T}^{\prime \prime}$ of $\mathbf{T}$ such that $\mathbf{T}=\mathbf{T}^{\prime \prime} \mathbf{T}^{\prime}$ (see $[\mathbf{8}$, Proposition 8.7]).

Lemma 7.2. $\mathbf{H}_{\Gamma}=\Gamma \mathbf{H}_{\Delta}$.

Proof. Consider the quotient $q: \mathbf{H}_{\Gamma} \rightarrow \mathbf{H}_{\Gamma} / \mathbf{H}_{\Delta}$. Since $\Gamma$ is Zariskidense in $\mathbf{H}_{\Gamma}, q(\Gamma)$ is Zariski-dense in $\mathbf{H}_{\Gamma} / \mathbf{H}_{\Delta}$. Since $\mathbf{H}_{\Gamma} / \mathbf{H}_{\Delta}$ is a finite group, $q(\Gamma)=\mathbf{H}_{\Gamma} / \mathbf{H}_{\Delta}$. Thus we have

$$
\Gamma \mathbf{H}_{\Delta}=\Gamma \mathbf{T}^{\prime} \ltimes \mathbf{U}_{\Gamma}=\mathbf{T}^{\prime \prime} \mathbf{T}^{\prime} \ltimes \mathbf{U}_{\Gamma}=\mathbf{H}_{\Gamma} .
$$

q.e.d.

Let $H_{\Gamma}=\mathbf{H}_{\Gamma}(\mathbb{R}), T^{\prime}=\mathbf{T}^{\prime}(\mathbb{R})$, and $T^{\prime \prime}=\mathbf{T}^{\prime \prime}(\mathbb{R})$. Then by Lemma 2.6 and $H_{G}=H_{\Delta}$, we have $H_{\Gamma}=T^{\prime} T^{\prime \prime} \ltimes G$. Hence we have $\Gamma \subset H_{\Gamma} \subset$ 
$\operatorname{Aut}(G) \ltimes G$. Since $\Delta$ is a lattice of $G$ and a finite index normal subgroup of $\Gamma, \Gamma$ is a discrete subgroup of $\operatorname{Aut}(G) \ltimes G$ and $G / \Gamma$ is compact and hence an infra-solvmanifold.

Theorem 7.3. Let $\Gamma$ be a torsion-free polycyclic group and $\Gamma \rightarrow \mathbf{H}_{\Gamma}$ be the algebraic hull of $\Gamma$. Suppose $\mathbf{H}_{\Gamma} / \mathbf{U}_{\Gamma}$ is diagonalizable. Let $\mathfrak{u}$ be the Lie algebra of $\mathbf{U}_{\Gamma}$. Let $\rho$ be the composition

$$
\Gamma \rightarrow \mathbf{H}_{\Gamma} \rightarrow \mathbf{H}_{\Gamma} / \mathbf{U}_{\Gamma} .
$$

Then we have a quasi-isomorphism

$$
\bigwedge \mathfrak{u}^{*} \rightarrow A^{*}\left(G / \Gamma, \mathcal{O}_{\rho}\right) .
$$

Proof. In this proof for a DGA $A$ with a group $G$-action, we denote $(A)^{G}$ the sub-DGA which consists of $G$-invariant elements of $A$. Consider decompositions $\mathbf{H}_{\Gamma}=\mathbf{T} \ltimes \mathbf{U}_{\Gamma}, \mathbf{H}_{\Delta}=\mathbf{T}^{\prime} \ltimes \mathbf{U}_{\Gamma}$ as above. Let $\left\{V_{\alpha}\right\}$ be the set of 1-dimensional representations of $\mathbf{T}$ for all characters $\alpha$ of T. Consider the DGA $\bigoplus_{\alpha} A^{*}(G) \otimes V_{\alpha}$ with the derivation $d$ given by

$$
d\left(\omega \otimes v_{\alpha}\right)=(d \omega) \otimes v_{\alpha} \quad \omega \in A^{*}(G), \quad v_{\alpha} \in V_{\alpha}
$$

and the products given by

$$
\left(\omega_{1} \otimes v_{\alpha}\right) \wedge\left(\omega_{2} \otimes v_{\beta}\right)=\left(\omega_{1} \wedge \omega_{2}\right) \otimes\left(v_{\alpha} \otimes v_{\beta}\right) .
$$

Then by the definition, we have

$$
A^{*}\left(G / \Gamma, \mathcal{O}_{\rho}\right)=\left(\bigoplus_{\alpha} A^{*}(G) \otimes V_{\alpha}\right)^{\Gamma} .
$$

Let $\left\{V_{\alpha^{\prime}}\right\}$ and $\left\{V_{\alpha^{\prime \prime}}\right\}$ be the sets of 1-dimensional representations of $\mathbf{T}^{\prime}$ and $\mathbf{T}^{\prime \prime}$ for all characters $\alpha^{\prime}$ of $\mathbf{T}^{\prime}$ and $\alpha^{\prime \prime}$ of $\mathbf{T}^{\prime \prime}$. By $\mathbf{T}=\mathbf{T}^{\prime} \mathbf{T}^{\prime \prime}$, we have $\left\{V_{\alpha}\right\}=\left\{V_{\alpha^{\prime}} \otimes V_{\alpha^{\prime \prime}}\right\}$. Then we have

$$
H^{*}\left(A^{*}\left(G / \Gamma, \mathcal{O}_{\rho}\right)\right)=H^{*}\left(\left(\bigoplus_{\alpha^{\prime}, \alpha^{\prime \prime}} A^{*}(G) \otimes\left(V_{\alpha^{\prime}} \otimes V_{\alpha^{\prime \prime}}\right)\right)^{\Gamma}\right) .
$$

Since $\Delta$ is a finite index normal subgroup of $\Gamma$, we have

$$
\begin{aligned}
& H^{*}\left(\left(\bigoplus_{\alpha^{\prime}, \alpha^{\prime \prime}} A^{*}(G) \otimes\left(V_{\alpha^{\prime}} \otimes V_{\alpha^{\prime \prime}}\right)\right)^{\Gamma}\right) \\
& \cong H^{*}\left(\left(\bigoplus_{\alpha^{\prime}, \alpha^{\prime \prime}} A^{*}(G) \otimes\left(V_{\alpha^{\prime}} \otimes V_{\alpha^{\prime \prime}}\right)\right)^{\Delta}\right)^{\Gamma / \Delta}
\end{aligned}
$$


Since $\Delta \subset \mathbf{T}^{\prime} \ltimes \mathbf{U}_{\Gamma}$, for a character $\alpha^{\prime \prime}$ of $\mathbf{T}^{\prime \prime}, \Delta$ acts trivially on $V_{\alpha^{\prime \prime}}$. Hence we have

$$
\begin{gathered}
H^{*}\left(\left(\bigoplus_{\alpha^{\prime}, \alpha^{\prime \prime}} A^{*}(G) \otimes\left(V_{\alpha^{\prime}} \otimes V_{\alpha^{\prime \prime}}\right)\right)^{\Delta}\right)^{\Gamma / \Delta} \\
=H^{*}\left(\bigoplus_{\alpha^{\prime \prime}}\left(\bigoplus_{\alpha^{\prime}} A^{*}(G) \otimes V_{\alpha^{\prime}}\right)^{\Delta} \otimes V_{\alpha^{\prime \prime}}\right)^{\Gamma / \Delta} .
\end{gathered}
$$

Since $\Delta$ is a lattice of $G$ and we assume that $\operatorname{Ad}(G)$ and $\operatorname{Ad}(\Delta)$ have the same Zariski-closure, by Corollary 6.2 we have

$$
H^{*}\left(\left(\bigoplus_{\alpha^{\prime}} A^{*}(G) \otimes V_{\alpha^{\prime}}\right)^{\Delta}\right) \cong H^{*}\left(\left(\bigoplus_{\alpha^{\prime}} A^{*}(G) \otimes V_{\alpha^{\prime}}\right)^{G}\right)
$$

By Lemmas 5.1 and 5.3, we have

$$
H^{*}\left(\left(\bigoplus_{\alpha^{\prime}} A^{*}(G) \otimes V_{\alpha^{\prime}}\right)^{G}\right) \cong H^{*}\left(\left(\left(\bigoplus_{\alpha^{\prime}} A^{*}(G) \otimes V_{\alpha^{\prime}}\right)^{G}\right)^{T^{\prime}}\right)
$$

Hence we have

$$
\begin{aligned}
& H^{*}\left(\bigoplus_{\alpha^{\prime \prime}}\left(\bigoplus_{\alpha^{\prime}} A^{*}(G) \otimes V_{\alpha^{\prime}}\right)^{\Delta} \otimes V_{\alpha^{\prime \prime}}\right)^{\Gamma / \Delta} \\
& \cong H^{*}\left(\bigoplus_{\alpha^{\prime \prime}}\left(\left(\bigoplus_{\alpha^{\prime}} A^{*}(G) \otimes V_{\alpha^{\prime}}\right)^{G}\right)^{T^{\prime}} \otimes V_{\alpha^{\prime \prime}}\right)^{\Gamma / \Delta} .
\end{aligned}
$$

Since $H_{\Delta}=T^{\prime} \ltimes G$, we have

$$
\begin{gathered}
H^{*}\left(\bigoplus_{\alpha^{\prime \prime}}\left(\left(\bigoplus_{\alpha^{\prime}} A^{*}(G) \otimes V_{\alpha^{\prime}}\right)^{G}\right)^{T^{\prime}} \otimes V_{\alpha^{\prime \prime}}\right)^{\Gamma / \Delta} \\
=H^{*}\left(\bigoplus_{\alpha^{\prime \prime}}\left(\bigoplus_{\alpha^{\prime}} A^{*}(G) \otimes V_{\alpha^{\prime}}\right)^{H_{\Delta}} \otimes V_{\alpha^{\prime \prime}}\right)^{\Gamma / \Delta} \\
\cong H^{*}\left(\left(\bigoplus_{\alpha^{\prime \prime}} \bigoplus_{\alpha^{\prime}} A^{*}(G) \otimes V_{\alpha^{\prime}} \otimes V_{\alpha^{\prime \prime}}\right)^{\Gamma H_{\Delta}}\right) .
\end{gathered}
$$


By Lemma 7.2, we have

$$
\begin{aligned}
H^{*}\left(\left(\bigoplus_{\alpha^{\prime \prime}} \bigoplus_{\alpha^{\prime}} A^{*}(G) \otimes V_{\alpha^{\prime}} \otimes V_{\alpha^{\prime \prime}}\right)^{\Gamma H_{\Delta}}\right) \\
=H^{*}\left(\left(\bigoplus_{\alpha^{\prime \prime}} \bigoplus_{\alpha^{\prime}} A^{*}(G) \otimes V_{\alpha^{\prime}} \otimes V_{\alpha^{\prime \prime}}\right)^{H_{\Gamma}}\right) .
\end{aligned}
$$

Since $H_{\Gamma}=T^{\prime} T^{\prime \prime} \ltimes G$, as above we have

$$
\begin{gathered}
H^{*}\left(\left(\bigoplus_{\alpha^{\prime \prime}} \bigoplus_{\alpha^{\prime}} A^{*}(G) \otimes V_{\alpha^{\prime}} \otimes V_{\alpha^{\prime \prime}}\right)^{H_{\Gamma}}\right) \\
=H^{*}\left(\left(\bigoplus_{\alpha^{\prime \prime}}\left(\left(\bigoplus_{\alpha^{\prime}} A^{*}(G) \otimes V_{\alpha^{\prime}}\right)^{G}\right)^{T^{\prime}} \otimes V_{\alpha^{\prime \prime}}\right)^{T^{\prime \prime}}\right) .
\end{gathered}
$$

Thus it is sufficient to show that the DGA

$$
\left(\bigoplus_{\alpha^{\prime \prime}}\left(\left(\bigoplus_{\alpha^{\prime}} A^{*}(G) \otimes V_{\alpha^{\prime}}\right)^{G}\right)^{T^{\prime}} \otimes V_{\alpha^{\prime \prime}}\right)^{T^{\prime \prime}}
$$

is isomorphic to $\bigwedge \mathfrak{u}^{*}$. By Lemma 5.3 we have

$$
\left(\bigoplus_{\alpha^{\prime \prime}}\left(\left(\bigoplus_{\alpha^{\prime}} A^{*}(G) \otimes V_{\alpha^{\prime}}\right)^{G}\right)^{T^{\prime}} \otimes V_{\alpha^{\prime \prime}}\right)^{T^{\prime \prime}} \cong\left(\bigoplus_{\alpha^{\prime \prime}} \bigwedge \mathfrak{u}^{*} \otimes V_{\alpha^{\prime \prime}}\right)^{T^{\prime \prime}}
$$

Now let $\bigwedge \mathfrak{u}^{*}=\bigoplus_{\beta} A_{\beta^{\prime \prime}}$ be the weight decomposition of $T^{\prime \prime}$ for characters $\beta^{\prime \prime}$ of $\mathbf{T}^{\prime \prime}$. Then we have

$$
\left(\bigoplus_{\alpha^{\prime \prime}} \bigwedge \mathfrak{u}^{*} \otimes V_{\alpha^{\prime \prime}}\right)^{T^{\prime \prime}}=\left(\bigoplus_{\beta^{\prime \prime}} \bigoplus_{\alpha^{\prime \prime}} A_{\beta^{\prime \prime}} \otimes V_{\alpha^{\prime \prime}}\right)^{T^{\prime \prime}}=\bigoplus_{\alpha^{\prime \prime}} A_{\left(\alpha^{\prime \prime}\right)^{-1}} \otimes V_{\alpha^{\prime \prime}}
$$

It is easily seen that

$$
\bigoplus_{\alpha^{\prime \prime}} A_{\left(\alpha^{\prime \prime}\right)^{-1}} \otimes V_{\alpha^{\prime \prime}} \cong \bigwedge \mathfrak{u}^{*}
$$

Hence the theorem follows.

q.e.d.

Obviously a solvmanifold $G / \Gamma$ is a infra-solvmanifold with polycyclic fundamental group $\Gamma$. Since $\mathbf{T}$ is the Zariski-closure of $\operatorname{Ad}_{s}(\Gamma)$ and diagonalizable, we have: 
Corollary 7.4. Let $G$ be a simply connected solvable Lie group with a lattice $\Gamma$ and $\mathbf{U}_{G}$ be the unipotent hull of $G$. Let $\mathfrak{u}$ be the Lie algebra of $\mathbf{U}_{G}$. Then we have a quasi-isomorphism

$$
\bigwedge \mathfrak{u}^{*} \rightarrow A^{*}\left(G / \Gamma, \mathcal{O}_{\operatorname{Ad}_{s \mid}}\right) .
$$

Thus $\bigwedge \mathfrak{u}^{*}$ is the minimal model of $A^{*}\left(G / \Gamma, \mathcal{O}_{\mathrm{Ad}_{\left.s\right|_{\Gamma}}}\right)$.

Consider the injection $\phi: A^{*}\left(\mathfrak{g}_{\mathbb{C}}, \operatorname{ad}_{s}\right) \rightarrow A^{*}\left(G / \Gamma, \mathcal{O}_{\mathrm{Ad}_{\left.s\right|_{\Gamma}}}\right)$. By Theorem 5.4, Proposition 6.1, and the corollary above, $\phi: A^{*}\left(\mathfrak{g}_{\mathbb{C}}, \operatorname{ad}_{s}\right) \rightarrow$ $A^{*}\left(G / \Gamma, \mathcal{O}_{\mathrm{Ad}_{s_{\mid}}}\right)$is a quasi-isomorphism. Hence we have:

Corollary 7.5. Let $G$ be a simply connected solvable Lie group with a lattice $\Gamma$. Then we have an isomorphism

$$
H^{*}\left(A^{*}\left(G / \Gamma, \mathcal{O}_{\operatorname{Ad}_{s \mid \Gamma}}\right)\right) \cong H^{*}\left(A^{*}\left(\mathfrak{g}_{\mathbb{C}}, \operatorname{ad}_{s}\right)\right) .
$$

We can apply this corollary to computations of the untwisted de Rham cohomology of solvmanifolds by invariant forms. We have an extension of Mostow's theorem (=Theorem 3.1) for the untwisted cohomology.

Corollary 7.6. Let $G$ be a simply connected solvable Lie group with a lattice $\Gamma$. Let $\mathbf{T}$ be the Zariski-closure of $\operatorname{Ad}_{s}(G)$ in $\operatorname{Aut}\left(\mathfrak{g}_{\mathbb{C}}\right)$. Denote $A_{\Gamma}$ a set of characters of $\mathbf{T}$ such that for $\alpha \in A_{\Gamma}$ the restriction of $\alpha \circ \mathrm{Ad}_{s}$ on $\Gamma$ is trivial. Consider the sub-DGA $\bigoplus_{\alpha \in A_{\Gamma}} \wedge \mathfrak{g}_{\mathbb{C}}^{*} \otimes V_{\alpha}$ of $A^{*}\left(\mathfrak{g}_{\mathbb{C}}, \operatorname{ad}_{s}\right)$. Then we have a quasi-isomorphism

$$
\left(\bigoplus_{\alpha \in A_{\Gamma}} \bigwedge \mathfrak{g}_{\mathbb{C}}^{*} \otimes V_{\alpha}\right)^{\mathbf{T}} \rightarrow \bigoplus_{\alpha \in A_{\Gamma}} \bigwedge \mathfrak{g}_{\mathbb{C}}^{*} \otimes V_{\alpha} \rightarrow A_{\mathbb{C}}^{*}(G / \Gamma) .
$$

Moreover, the DGA $\left(\bigoplus_{\alpha \in A_{\Gamma}} \wedge \mathfrak{g}_{\mathbb{C}}^{*} \otimes V_{\alpha}\right)^{\mathbf{T}}$ is a sub-DGA of $\bigwedge \mathfrak{u}^{*}$.

Proof. Since we can consider $A_{\mathbb{C}}^{*}(G / \Gamma)=A^{*}\left(G / \Gamma, E_{1}\right)$ for the trivial character $\mathbf{1}, A_{\mathbb{C}}^{*}(G / \Gamma)$ is a sub-DGA of $A^{*}\left(G / \Gamma, \mathcal{O}_{\operatorname{Ad}_{\left.s\right|_{\Gamma}}}\right)$. Then we have

$$
\phi^{-1}\left(A_{\mathbb{C}}^{*}(G / \Gamma)\right)=\bigoplus_{\alpha \in A_{\Gamma}} \bigwedge \mathfrak{g}_{\mathbb{C}}^{*} \otimes V_{\alpha} .
$$

Since we define $A^{*}\left(G / \Gamma, \mathcal{O}_{\operatorname{Ad}_{\left.s\right|_{\Gamma}}}\right)=\bigoplus A^{*}\left(G / \Gamma, E_{\left.\alpha \circ A_{s \mid}\right|_{\Gamma}}\right)$ as a direct sum of cochain complexes and $\phi: A^{*}\left(\mathfrak{g}_{\mathbb{C}}, \operatorname{ad}_{s}\right) \rightarrow A^{*}\left(G / \Gamma, \mathcal{O}_{\operatorname{Ad}_{\left.s\right|_{\Gamma}}}\right)$ is a quasi-isomorphism by Corollary 7.5, the restriction $\phi: \phi^{-1}\left(A_{\mathbb{C}}^{*}(G / \Gamma)\right) \rightarrow$ $A_{\mathbb{C}}^{*}(G / \Gamma)$ is also a quasi-isomorphism. By Lemma 5.1, the inclusion

$$
\left(\bigoplus_{\alpha \in A_{\Gamma}} \bigwedge \mathfrak{g}_{\mathbb{C}}^{*} \otimes V_{\alpha}\right)^{\mathbf{T}} \rightarrow \bigoplus_{\alpha \in A_{\Gamma}} \bigwedge \mathfrak{g}_{\mathbb{C}}^{*} \otimes V_{\alpha}
$$


is a quasi-isomorphism. By Lemma 5.2, $\left(\bigoplus_{\alpha \in A_{\Gamma}} \wedge \mathfrak{g}_{\mathbb{C}}^{*} \otimes V_{\alpha}\right)^{\mathbf{T}}$ is a subDGA of $\bigwedge \mathfrak{u}^{*}$. Hence the corollary follows.

\section{Formality and hard Lefschetz properties}

In [15], Hasegawa proved the following theorem.

Theorem 8.1. ([15]) Consider a DGA $\bigwedge \mathfrak{n}^{*}$ which is the dual of a nilpotent Lie algebra $\mathfrak{n}$. Then $\bigwedge \mathfrak{n}^{*}$ is formal if and only if $\mathfrak{n}$ is abelian.

By Hasegawa's theorem, Theorem 5.4, Proposition 2.7, and Corollary 7.4 , we have the following theorem.

Theorem 8.2. Let $G$ be a simply connected solvable Lie group. Then the following conditions are equivalent:

(A) The DGA $A^{*}\left(\mathfrak{g}_{\mathbb{C}}, a_{s}\right)$ is formal.

(B) $\mathbf{U}_{G}$ is abelian.

(C) $G=\mathbb{R}^{n} \ltimes_{\phi} \mathbb{R}^{m}$ such that the action $\phi: \mathbb{R}^{n} \rightarrow \operatorname{Aut}\left(\mathbb{R}^{m}\right)$ is semisimple.

Moreover, suppose $G$ has a lattice $\Gamma$. Then the above three conditions are equivalent to the following condition:

(D) $A^{*}\left(G / \Gamma, \mathcal{O}_{\operatorname{Ad}_{s \mid \Gamma}}\right)$ is formal.

In [6], Benson and Gordon proved:

Theorem 8.3. ([6]; see also [13, Section 4.6.4]) Consider a DGA $\bigwedge \mathfrak{n}^{*}$ which is the cochain complex of the dual of a nilpotent Lie algebra $\mathfrak{n}$. Suppose we have $[\omega] \in H^{2}\left(\bigwedge \mathfrak{n}^{*}\right)$ such that $[\omega]^{n} \neq 0$ where $2 n=\operatorname{dim} \mathfrak{n}$. Then for any $0 \leq i \leq n$ the linear operator

$$
[\omega]^{n-i} \wedge: H^{i}\left(\bigwedge \mathfrak{n}^{*}\right) \rightarrow H^{2 n-i}\left(\bigwedge \mathfrak{n}^{*}\right)
$$

is an isomorphism if and only if $\mathfrak{n}$ is abelian.

By this theorem, we have:

Theorem 8.4. Let $G$ be a simply connected solvable Lie group. Suppose $\operatorname{dim} G=2 n$ and $G$ has an $G$-invariant symplectic form $\omega$. Then the following conditions are equivalent:

(A)

$$
[\omega]^{n-i} \wedge: H^{i}\left(A^{*}\left(\mathfrak{g}_{\mathbb{C}}, \operatorname{ad}_{s}\right)\right) \rightarrow H^{2 n-i}\left(A^{*}\left(\mathfrak{g}_{\mathbb{C}}, \operatorname{ad}_{s}\right)\right)
$$

is an isomorphic for any $i \leq n$.

(B) $\mathbf{U}_{G}$ is abelian.

(C) $G=\mathbb{R}^{n} \ltimes_{\phi} \mathbb{R}^{m}$ such that the action $\phi: \mathbb{R}^{n} \rightarrow \operatorname{Aut}\left(\mathbb{R}^{m}\right)$ is semisimple.

Suppose $G$ has a lattice $\Gamma$ and $G / \Gamma$ has a symplectic form (not necessarily $G$-invariant) $\omega$. Then the conditions $(B)$ and $(C)$ are equivalent 
to the following condition:

$(D)$

$$
[\omega]^{n-i} \wedge: H^{i}\left(A^{*}\left(G / \Gamma, \mathcal{O}_{\operatorname{Ad}_{\left.s\right|_{\Gamma}}}\right)\right) \rightarrow H^{2 n-i}\left(A^{*}\left(G / \Gamma, \mathcal{O}_{\operatorname{Ad}_{\left.s\right|_{\Gamma}}}\right)\right)
$$

is an isomorphism for any $i \leq n$.

For infra-solvmanifolds, by Theorem 7.3 and Proposition 2.7 we have:

Theorem 8.5. Let $M$ be a infra-solvmanifold with the torsion-free polycyclic fundamental group $\Gamma$, and let $\Gamma \rightarrow \mathbf{H}_{\Gamma}$ be the algebraic hull of $\Gamma$. Suppose $\mathbf{H}_{\Gamma} / \mathbf{U}_{\Gamma}$ is diagonalizable. Let $\rho$ be the composition

$$
\Gamma \rightarrow \mathbf{H}_{\Gamma} \rightarrow \mathbf{H}_{\Gamma} / \mathbf{U}_{\Gamma} .
$$

Then following conditions are equivalent:

(A) $A^{*}\left(M, \mathcal{O}_{\rho}\right)$ is formal.

(B) $\mathbf{U}_{\Gamma}$ is abelian.

(C) $M$ is finitely covered by a solvmanifold $G / \Gamma$ such that $G=\mathbb{R}^{n} \ltimes_{\phi} \mathbb{R}^{m}$ with a semi-simple action $\phi: \mathbb{R}^{n} \rightarrow \operatorname{Aut}\left(\mathbb{R}^{m}\right)$, and $\Gamma$ is a lattice of $G$.

If $\operatorname{dim} M=2 n$ and $M$ has a symplectic form $\omega$, the conditions $(A)$, $(B)$, and $(C)$ are equivalent to the following condition:

$$
[\omega]^{n-i} \wedge: H^{i}\left(A^{*}\left(M, \mathcal{O}_{\rho}\right)\right) \rightarrow H^{2 n-i}\left(A^{*}\left(M, \mathcal{O}_{\rho}\right)\right)
$$

is an isomorphism for any $i \leq n$.

\section{Examples and remarks}

Let $G$ be a simply connected solvable Lie group with a lattice $\Gamma$. Suppose $\mathbf{U}_{G}$ is abelian. In $[\mathbf{1 9}]$ the author showed that $G / \Gamma$ is formal and if $G / \Gamma$ has a symplectic form, then $G / \Gamma$ is hard Lefschetz. But the converses of these results are not true. See the following examples.

Example 1. ([27]) We consider a 8-dimensional solvable Lie group $G=G_{1} \times \mathbb{R}$ such that: $G_{1}$ is the matrix group as

$$
\left\{\left(\begin{array}{ccccccc}
e^{a_{1} t} & 0 & 0 & 0 & 0 & e^{-a_{3} t} x_{2} & y_{1} \\
0 & e^{a_{2} t} & 0 & e^{-a_{1} t} x_{3} & 0 & 0 & y_{2} \\
0 & 0 & e^{a_{3} t} & 0 & e^{-a_{2} t} x_{1} & 0 & y_{3} \\
0 & 0 & 0 & e^{-a_{1} t} & 0 & 0 & x_{1} \\
0 & 0 & 0 & 0 & e^{-a_{2} t} & 0 & x_{2} \\
0 & 0 & 0 & 0 & 0 & e^{-a_{3} t} & x_{3} \\
0 & 0 & 0 & 0 & 0 & 0 & 1
\end{array}\right): t, x_{i}, y_{i} \in \mathbb{R}\right\}
$$

where $a_{1}, a_{2}, a_{3}$ are distinct real numbers such that $a_{1}+a_{2}+a_{3}=0$.

Let $\mathfrak{g}$ be the Lie algebra of $G$, and $\mathfrak{g}^{*}$ the dual of $\mathfrak{g}$. The cochain complex $\left(\bigwedge \mathfrak{g}^{*}, d\right)$ is generated by a basis $\left\{\alpha, \beta, \zeta_{i}, \eta_{i}\right\}$ of $\mathfrak{g}^{*}$ such that:

$$
d \alpha=0, d \beta=0,
$$




$$
\begin{gathered}
d \zeta_{i}=a_{i} \alpha \wedge \zeta_{i}, \\
d \eta_{1}=-a_{1} \alpha \wedge \eta_{1}-\zeta_{2} \wedge \zeta_{3}, \\
d \eta_{2}=-a_{2} \alpha \wedge \eta_{2}-\zeta_{3} \wedge \zeta_{1}, \\
d \eta_{3}=-a_{3} \alpha \wedge \eta_{3}-\zeta_{1} \wedge \zeta_{2} .
\end{gathered}
$$

In [27] Sawai showed that for some $a_{1}, a_{2}, a_{3}, G$ has a lattice $\Gamma$ and $G / \Gamma$ satisfies formality and has a $G$-invariant symplectic form

$$
\omega=\alpha \wedge \beta+p\left(\zeta_{1} \wedge \eta_{1}-\zeta_{2} \wedge \eta_{2}\right)+q\left(-\zeta_{2} \wedge \eta_{2}+\zeta_{3} \wedge \eta_{3}\right)
$$

satisfying the hard Lefschetz property where $p q \neq 0$ and $p+q \neq 0$. We have

$$
\operatorname{Ad}_{s}(G)=\left\{\left(\begin{array}{cccccccc}
e^{a_{1} t} & 0 & 0 & 0 & 0 & 0 & 0 & 0 \\
0 & e^{a_{2} t} & 0 & 0 & 0 & 0 & 0 & 0 \\
0 & 0 & e^{a_{3} t} & 0 & 0 & 0 & 0 & 0 \\
0 & 0 & 0 & e^{-a_{1} t} & 0 & 0 & 0 & 0 \\
0 & 0 & 0 & 0 & e^{-a_{2} t} & 0 & 0 & 0 \\
0 & 0 & 0 & 0 & 0 & e^{-a_{3} t} & 0 & 0 \\
0 & 0 & 0 & 0 & 0 & 0 & 1 & 0 \\
0 & 0 & 0 & 0 & 0 & 0 & 0 & 1
\end{array}\right): t \in \mathbb{R}\right\} .
$$

Let $\mathbf{T}$ be the Zariski closure of $\operatorname{Ad}_{s}(G)$. Then for some characters $\alpha_{1}, \alpha_{2}, \alpha_{3}$ of $\mathbf{T}$, the cochain complexes $\left(\bigwedge \mathfrak{g}^{*} \otimes V_{\alpha_{i}}, d_{\alpha_{i}}\right)$ are given by:

$$
d_{\alpha_{i}}\left(v_{\alpha_{i}}\right)=-a_{i} \alpha \otimes v_{\alpha_{i}}
$$

for $v_{\alpha_{i}} \in V_{\alpha_{i}}$.

We have

$$
\begin{gathered}
d_{\alpha_{2}}\left(\zeta_{2} \otimes v_{\alpha_{2}}\right)=a_{2} \alpha \wedge \zeta_{2} \otimes v_{\alpha_{2}}+\zeta_{2} \wedge a_{2} \alpha \otimes v_{\alpha_{2}}=0, \\
d_{\alpha_{3}}\left(\zeta_{3} \otimes v_{\alpha_{3}}\right)=a_{3} \alpha \wedge \zeta_{3} \otimes v_{\alpha_{3}}+\zeta_{3} \wedge a_{3} \alpha \otimes v_{\alpha_{3}}=0, \\
d_{\alpha_{2} \alpha_{3}}\left(\eta_{1} \otimes v_{\alpha_{2} \alpha_{3}}\right) \\
=-\left(a_{1}+a_{2}+a_{3}\right) \alpha \wedge \eta_{1} \otimes v_{\alpha_{2} \alpha_{3}}-\zeta_{2} \wedge \zeta_{3} \otimes v_{\alpha_{2} \alpha_{3}} \\
=-\zeta_{2} \wedge \zeta_{3} \otimes v_{\alpha_{2} \alpha_{3}} .
\end{gathered}
$$

Hence in $H^{2}\left(\bigwedge \mathfrak{g}_{\mathbb{C}}^{*} \otimes V_{\alpha_{2} \alpha_{3}}\right)$,

$$
\left[\zeta_{2} \otimes v_{\alpha_{2}}\right] \cdot\left[\zeta_{3} \otimes v_{\alpha_{3}}\right]=0
$$

and we have the Massey triple product

$$
\left\langle\left[\zeta_{2} \otimes v_{\alpha_{2}}\right],\left[\zeta_{3} \otimes v_{\alpha_{3}}\right],\left[\zeta_{3} \otimes v_{\alpha_{3}}\right]\right\rangle=\left[\eta_{1} \wedge \zeta_{3} \otimes v_{\alpha_{2} \alpha_{3}^{2}}\right]
$$

in the quotient of

by

$$
H^{2}\left(\bigwedge \mathfrak{g}_{\mathbb{C}}^{*} \otimes V_{\alpha_{2} \alpha_{3}^{2}}\right)
$$

$$
\left(\left[\zeta_{2} \otimes v_{\alpha_{2}}\right] \cdot H^{1}\left(\bigwedge \mathfrak{g}_{\mathbb{C}}^{*} \otimes V_{\alpha_{3}^{2}}\right)+\left[\zeta_{3} \otimes v_{\alpha_{3}}\right] \cdot H^{1}\left(\bigwedge \mathfrak{g}_{\mathbb{C}}^{*} \otimes V_{\alpha_{2} \alpha_{3}}\right)\right) .
$$


This Massey product is not zero. Hence the DGA $\bigoplus_{\alpha} \wedge \mathfrak{g}^{*} \otimes V_{\alpha}$ has a non-zero Massey product and it is not formal.

Remark 9.1. In [22], Narkawicz gave examples of complements $X$ of hyperplane arrangements which are formal, but for some diagonal representations of $\pi_{1}(X, x)$ the DGA $A^{*}\left(X, \mathcal{O}_{\rho}\right)$ is non-formal.

We have $d_{\alpha_{1}}\left(\zeta_{1} \otimes v_{\alpha_{1}}\right)=0$ and the cohomology class $\left[\zeta_{1} \otimes v_{\alpha_{1}}\right] \in$ $H^{1}\left(\bigwedge \mathfrak{g}_{\mathbb{C}}^{*} \otimes V_{\alpha_{1}}\right)$ is not zero. We have

$$
\begin{aligned}
\omega^{3}=-6 p(p+q) \alpha & \wedge \beta \wedge \zeta_{1} \wedge \eta_{1} \wedge \zeta_{2} \wedge \eta_{2} \\
-6(p+q) q \alpha & \wedge \beta \wedge \zeta_{2} \wedge \eta_{2} \wedge \zeta_{3} \wedge \eta_{3} \\
+6 p q \alpha & \wedge \beta \wedge \zeta_{1} \wedge \eta_{1} \wedge \zeta_{3} \wedge \eta_{3} \\
& -6 p q(p+q) \zeta_{1} \wedge \eta_{1} \wedge \zeta_{2} \wedge \eta_{2} \wedge \zeta_{3} \wedge \eta_{3},
\end{aligned}
$$

and

$$
\omega^{3} \wedge \zeta_{1} \otimes v_{\alpha_{1}}=-6(p+q) q \alpha \wedge \beta \wedge \zeta_{1} \wedge \zeta_{2} \wedge \eta_{2} \wedge \zeta_{3} \wedge \eta_{3} \otimes v_{\alpha_{1}} .
$$

Otherwise we have

$d_{\alpha_{1}}\left(\alpha \wedge \beta \wedge \zeta_{1} \wedge \eta_{1} \wedge \eta_{2} \wedge \eta_{3} \otimes v_{\alpha_{1}}\right)=-\alpha \wedge \beta \wedge \zeta_{1} \wedge \zeta_{2} \wedge \eta_{2} \wedge \zeta_{3} \wedge \eta_{3} \otimes v_{\alpha_{1}}$. Hence $[\omega]^{3} \wedge\left(\left[\zeta_{1} \otimes v_{\alpha_{1}}\right]\right)=0$ and the operator $[\omega]^{3} \wedge$ is not injective.

Theorem 9.1. For $G / \Gamma$, the $\left.D G A A^{*}\left(G / \Gamma, \mathcal{O}_{A d_{s_{\Gamma}}}\right)\right)$ is not formal and the linear operator

$$
[\omega]^{3} \wedge: H^{1}\left(A^{*}\left(G / \Gamma, \mathcal{O}_{\mathrm{Ad}_{s \mid}}\right)\right) \rightarrow H^{7}\left(A^{*}\left(G / \Gamma, \mathcal{O}_{\mathrm{Ad}_{s \mid \Gamma}}\right)\right)
$$

is not an isomorphism. Thus $\mathbf{U}_{G}$ is not abelian. In particular $G / \Gamma$ is not Kähler.

As shown above, compared with untwisted versions, formality and the hard Lefschetz properties of the DGA $A^{*}\left(G / \Gamma, \mathcal{O}_{\operatorname{Ad}_{s_{\mid}}}\right)$are useful criteria for determining that formal and hard Lefschetz solvmanifolds to be not Kähler. But we have a non-Kähler symplectic solvmanifold such that $A^{*}\left(G / \Gamma, \mathcal{O}_{\operatorname{Ad}_{\left.s\right|_{\Gamma}}}\right)$ is formal and hard Lefschetz. In [1] Arapura showed that for a simply connected solvable Lie group $G$ with a lattice $\Gamma$, if a solvmanifold $G / \Gamma$ admits a Kähler structure then $\Gamma$ is virtually abelian. In [3] it was proved that a lattice of a simply connected solvable Lie group $G$ is virtually nilpotent if and only if $G$ is type (I), i.e. for any $g \in G$ all the eigenvalues of $\operatorname{Ad}_{g}$ have absolute value 1 . Thus by Theorem 8.2 and 8.4, we have:

Corollary 9.2. Let $G=\mathbb{R}^{n} \ltimes_{\phi} \mathbb{R}^{m}$ such that the action $\phi: \mathbb{R}^{n} \rightarrow$ Aut $\left(\mathbb{R}^{m}\right)$ is semi-simple. Suppose $G$ is not type (I) and has a lattice $\Gamma$. Then $A^{*}\left(G / \Gamma, \mathcal{O}_{\mathrm{Ad}_{\left.s\right|_{\Gamma}}}\right)$ is formal but $G / \Gamma$ has no Kähler structure. If $G / \Gamma$ has a symplectic form $\omega$, then the operator

$$
[\omega]^{n-i} \wedge: H^{i}\left(A^{*}\left(G / \Gamma, \mathcal{O}_{\operatorname{Ad}_{\left.s\right|_{\Gamma}}}\right)\right) \rightarrow H^{2 n-i}\left(A^{*}\left(G / \Gamma, \mathcal{O}_{\operatorname{Ad}_{\left.s\right|_{\Gamma}}}\right)\right)
$$


is an isomorphism for any $i \leq n$ where $\operatorname{dim} G=2 n$.

We give complex examples.

Example 2. ([21])

Let $G=\mathbb{C} \ltimes_{\phi} \mathbb{C}^{2}$ with $\phi(x)=\left(\begin{array}{cc}e^{x} & 0 \\ 0 & e^{-x}\end{array}\right)$. Then $G$ has an invariant symplectic form. In $[\mathbf{2 1}]$, it was shown that $G$ has a lattice $\Gamma$. Thus $G / \Gamma$ is a non-Kähler complex solvmanifold but $A^{*}\left(G / \Gamma, \mathcal{O}_{\mathrm{Ad}_{\left.s\right|_{\Gamma}}}\right)$ is formal and hard Lefschetz.

\section{On isomorphism $H^{*}(G / \Gamma, \mathbb{C}) \cong H^{*}\left(\mathfrak{g}_{\mathbb{C}}\right)$}

Let $G$ be a simply connected solvable Lie group with a lattice $\Gamma$, and let $\mathfrak{g}$ be the Lie algebra of $G$. We give new criteria for the isomorphism $H^{*}(G / \Gamma, \mathbb{C}) \cong H^{*}\left(\mathfrak{g}_{\mathbb{C}}\right)$ to hold by using Corollary 7.4. Take a basis $X_{1}, \ldots, X_{n}$ of $\mathfrak{g}_{\mathbb{C}}$ such that $\operatorname{Ad}_{s}$ is represented by diagonal matrices as $\operatorname{Ad}_{s g}=\operatorname{diag}\left(\alpha_{1}(g), \ldots, \alpha_{n}(g)\right)$. For $\left\{i_{1}, \ldots, i_{p}\right\} \subset\{1, \ldots, n\}$ write $\alpha_{i_{1}, \ldots, i_{p}}$ as the product of characters $\alpha_{i_{1}}, \ldots, \alpha_{i_{p}}$.

Corollary 10.1. Let $G$ be a simply connected solvable Lie group with a lattice $\Gamma$, and let $\mathfrak{g}$ be the Lie algebra of $G$. Suppose $(G, \Gamma)$ satisfies the following condition:

$\left(C_{G, \Gamma}\right):$ For any $\left\{i_{1}, \ldots, i_{p}\right\} \subset\{1, \ldots, n\}$, if the character $\alpha_{i_{1}, \ldots, i_{p}}$ is non-trivial, then the restriction of $\alpha_{i_{1}, \ldots, i_{p} \mid \Gamma}$ on $\Gamma$ is also non-trivial.

Then an isomorphism $H^{*}(G / \Gamma, \mathbb{C}) \cong H^{*}\left(\mathfrak{g}_{\mathbb{C}}\right)$ holds.

Proof. Let $x_{1}, \ldots, x_{n}$ be a basis of $\mathfrak{g}_{\mathbb{C}}^{*}$ which is dual to $X_{1}, \ldots, X_{n}$. Consider the DGA $\left(\bigoplus_{\alpha \in A_{\Gamma}} \wedge \mathfrak{g}_{\mathbb{C}}^{*} \otimes V_{\alpha}\right)^{\mathbf{T}}$ as Corollary 7.4. By $\operatorname{Ad}_{s g}^{*}$. $x_{i}=\alpha_{i}(g)^{-1} x_{i}$ we have

$$
\begin{aligned}
& \left(\bigoplus_{\alpha \in A_{\Gamma}} \wedge \mathfrak{g}_{\mathbb{C}}^{*} \otimes V_{\alpha}\right)^{\mathbf{T}} \\
= & \left\langle x_{i_{1}} \wedge \cdots \wedge x_{i_{p}} \otimes v_{\alpha_{i_{1}, \ldots, i_{p}}} \mid \begin{array}{c}
1 \leq i_{1}<i_{2}<\cdots<i_{p} \leq n, \\
\text { the restriction of } \alpha_{i_{1}, \ldots, i_{p}} \text { on } \Gamma \text { is trivial }
\end{array}\right\rangle
\end{aligned}
$$

as the proof of Lemma 5.2. Suppose $(G, \Gamma)$ satisfies the condition $\left(C_{G, \Gamma}\right)$.

Then we have

$$
\left(\bigoplus_{\alpha \in A_{\Gamma}} \bigwedge \mathfrak{g}_{\mathbb{C}}^{*} \otimes V_{\alpha}\right)^{\mathbf{T}}=\left(\bigwedge \mathfrak{g}_{\mathbb{C}}^{*}\right)^{\mathbf{T}} .
$$

Hence by Corollary 7.4, we have an isomorphism

$$
H^{*}\left(\left(\bigwedge \mathfrak{g}_{\mathbb{C}}^{*}\right)^{\mathbf{T}}\right) \cong H^{*}(G / \Gamma, \mathbb{C}) .
$$


This implies that the inclusion $\bigwedge\left(\mathfrak{g}_{\mathbb{C}}\right)^{*} \subset A_{\mathbb{C}}^{*}(G / \Gamma)$ induces an isomorphism

$$
H^{*}\left(\mathfrak{g}_{\mathbb{C}}\right) \cong H^{*}(G / \Gamma, \mathbb{C})
$$

q.e.d.

Remark 10.1. We have examples such that we can apply this corollary but cannot use Mostow's theorem (=Theorem 3.1 ).

Example 3. Let $G=\mathbb{R} \ltimes_{\phi} \mathbb{R}^{2}$ with $\phi(t)=\left(\begin{array}{cc}\cos \pi t & -\sin \pi t \\ \sin \pi t & \cos \pi t\end{array}\right)$. Then $G$ has a lattice $\Gamma=\mathbb{Z} \ltimes \mathbb{Z}^{2}$. In this case $G$ is not completely solvable and $(G, \Gamma)$ does not satisfies the Mostow's condition. But diagonalization of $\operatorname{Ad}_{s}$ is given by $\operatorname{Ad}_{s}(t, x, y)=\operatorname{diag}\left(1, e^{\pi t \sqrt{-1}}, e^{-\pi t \sqrt{-1}}\right)$ and hence $(G, \Gamma)$ satisfies the condition $\left(C_{, G, \Gamma}\right)$. Thus we have an isomorphism $H^{*}\left(\mathfrak{g}_{\mathbb{C}}\right) \cong H^{*}(G / \Gamma, \mathbb{C})$.

For a character $\alpha$ of $G$, if the restriction of $\alpha$ on $\Gamma$ is trivial, then the image $\alpha(G)=\alpha(G / \Gamma)$ is compact and hence $\alpha$ is a unitary character. Hence the above corollary reduces to the following corollary.

Corollary 10.2. Let $G$ be a simply connected solvable Lie group with a lattice $\Gamma$, and let $\mathfrak{g}$ be the Lie algebra of $G$. Suppose $G$ satisfies the following condition:

$\left(D_{G}\right):$ For each $\left\{i_{1}, \ldots, i_{p}\right\} \subset\{1, \ldots, n\}$ the character $\alpha_{i_{1}, \ldots, i_{p}}$ is not a non-trivial unitary character.

Then an isomorphism $H^{*}(G / \Gamma, \mathbb{C}) \cong H^{*}\left(\mathfrak{g}_{\mathbb{C}}\right)$ holds.

Since the condition $\left(D_{G}\right)$ does not concern $\Gamma$, this corollary is more useful than the earlier corollary. Clearly a completely solvable Lie group satisfies the condition $\left(D_{G}\right)$. Hence this corollary is a generalization of Hattori's result in $[\mathbf{1 8}]$.

Example 4. Let $G=\mathbb{R}^{s} \ltimes_{\phi}\left(\mathbb{R}^{s} \times \mathbb{C}\right)$ such that

$$
\phi\left(t_{1}, \ldots, t_{s}\right)=\left(\begin{array}{ccccc}
e^{t_{1}} & 0 & \cdots & 0 & 0 \\
0 & \ddots & \ddots & \vdots & \vdots \\
\vdots & \ddots & e^{t_{s}} & 0 & 0 \\
0 & \cdots & 0 & e^{-\frac{1}{2}\left(t_{1}+\cdots t_{s}\right)} \cos \varphi & -e^{-\frac{1}{2}\left(t_{1}+\cdots t_{s}\right)} \sin \varphi \\
0 & \cdots & 0 & e^{-\frac{1}{2}\left(t_{1}+\cdots t_{s}\right)} \sin \varphi & e^{-\frac{1}{2}\left(t_{1}+\cdots t_{s}\right)} \cos \varphi
\end{array}\right) \text {, }
$$

where $\varphi=c_{1} t_{1}+\cdots+c_{s} t_{s}$. Then a diagonalization of $\operatorname{Ad}_{s}$ is given by

$$
\operatorname{Ad}_{s}=\operatorname{diag}\left(e^{t_{1}}, \ldots, e^{t_{s}}, e^{-\frac{1}{2}\left(t_{1}+\cdots t_{s}\right)+\varphi \sqrt{-1}}, e^{-\frac{1}{2}\left(t_{1}+\cdots t_{s}\right)-\varphi \sqrt{-1}}, 1, \ldots, 1\right) .
$$

By this, $G$ satisfies the condition $\left(D_{G}\right)$ for any $S$.

Proposition 10.3. For any lattice $\Gamma$, we have $b_{p}(G / \Gamma)=b_{2 s+2-p}(G / \Gamma)=$ ${ }_{s} \mathrm{C}_{p}$ for $1 \leq p \leq s$ and $b_{s+1}(G / \Gamma)=0$. 
Proof. For a coordinate $\left(t_{1}, \ldots, t_{s}, x_{1}, \ldots, x_{s}, z\right) \in \mathbb{R}^{s} \ltimes_{\phi}\left(\mathbb{R}^{s} \times \mathbb{C}\right)$, the cochain complex $\bigwedge \mathfrak{g}_{\mathbb{C}}^{*}$ is generated by

$$
\begin{aligned}
& \left\{d t_{1}, \ldots, d t_{s}, e^{-t_{1}} d x_{1}, \ldots, e^{-t_{s}} d x_{s},\right. \\
& \left.e^{\frac{1}{2}\left(t_{1}+\cdots t_{s}\right)-\varphi \sqrt{-1}} d z, e^{\frac{1}{2}\left(t_{1}+\cdots t_{s}\right)+\varphi \sqrt{-1}} d \bar{z}\right\} .
\end{aligned}
$$

Since $G$ satisfies the condition $\left(D_{p, G}\right)$, we have an isomorphism

$$
H^{p}(G / \Gamma, \mathbb{C}) \cong H^{*}\left(\left(\bigwedge^{p} \mathfrak{g}_{\mathbb{C}}^{*}\right)^{\mathbf{T}}\right)
$$

We have

$$
\left(\bigwedge^{p} \mathfrak{g}_{\mathbb{C}}^{*}\right)^{\mathbf{T}}=\left\langle d t_{i_{1}} \wedge \cdots \wedge d t_{i_{p}} \mid 1 \leq i_{1}<\cdots<i_{p} \leq s\right\rangle
$$

for $1 \leq p \leq s$ and $\left(\bigwedge^{s+1} \mathfrak{g}_{\mathbb{C}}^{*}\right)^{\mathbf{T}}=0$. Since the restriction of the derivation on $\left(\bigwedge^{p} \mathfrak{g}_{\mathbb{C}}^{*}\right)^{\mathbf{T}}$ is 0 for $1 \leq p \leq s+1$, we have

$$
H^{*}\left(\left(\bigwedge^{p} \mathfrak{g}_{\mathbb{C}}^{*}\right)^{\mathbf{T}}\right) \cong\left\langle d t_{i_{1}} \wedge \cdots \wedge d t_{i_{p}} \mid 1 \leq i_{1}<\cdots<i_{p} \leq s\right\rangle .
$$

By the Poincaré duality, we have the proposition.

q.e.d.

We can construct a lattice of $G$ using number theory. Let $K$ be a finite extension field of $\mathbb{Q}$ with the degree $s+2(s>0)$. Suppose $K$ admits embeddings $\sigma_{1}, \ldots, \sigma_{s}, \sigma_{s+1}, \sigma_{s+2}$ into $\mathbb{C}$ such that $\sigma_{1}, \ldots, \sigma_{s}$ are real embeddings and $\sigma_{s+1}, \sigma_{s+2}$ are complex ones satisfying $\sigma_{s+1}=\bar{\sigma}_{s+2}$. We can choose $K$ admitting such embeddings (see [25]). Denote $\mathcal{O}_{K}$ the ring of algebraic integers of $K, \mathcal{O}_{K}^{*}$ the group of units in $\mathcal{O}_{K}$, and

$$
\mathcal{O}_{K}^{*+}=\left\{a \in \mathcal{O}_{K}^{*}: \sigma_{i}>0 \text { for all } 1 \leq i \leq s\right\} .
$$

Define $\sigma: \mathcal{O}_{K} \rightarrow \mathbb{R}^{s} \times \mathbb{C}$ by

$$
\sigma(a)=\left(\sigma_{1}(a), \ldots, \sigma_{s}(a), \sigma_{s+1}(a)\right)
$$

for $a \in \mathcal{O}_{K}$. Then the image $\sigma\left(\mathcal{O}_{K}\right)$ is a lattice in $\mathbb{R}^{s} \times \mathbb{C}$. We denote

$$
\begin{aligned}
& \sigma(a) \cdot \sigma(b) \\
& \quad=\left(\sigma_{1}(a) \sigma_{1}(b), \ldots, \sigma_{s}(a) \sigma_{s}(b), \sigma_{s+1}(a) \sigma_{s+1}(b), \ldots, \sigma_{s+t}(a) \sigma_{s+t}(b)\right)
\end{aligned}
$$

for $a, b \in \mathcal{O}_{K}$. Define $l: \mathcal{O}_{K}^{*+} \rightarrow \mathbb{R}^{s+1}$ by

$$
l(a)=\left(\log \left|\sigma_{1}(a)\right|, \ldots, \log \left|\sigma_{s}(a)\right|, 2 \log \left|\sigma_{s+1}(a)\right|\right)
$$

for $a \in \mathcal{O}_{K}^{*+}$. Then by Dirichlet's units theorem, $l\left(\mathcal{O}_{K}^{*+}\right)$ is a lattice in the vector space $L=\left\{x \in \mathbb{R}^{s+1} \mid \sum_{i=1}^{s+1} x_{i}=0\right\}$. By this we have a 
geometrical representation of the semi-direct product $l\left(\mathcal{O}_{K}^{*+}\right) \ltimes_{\phi} \sigma\left(\mathcal{O}_{K}\right)$ with

$$
\phi\left(t_{1}, \ldots, t_{s+1}\right)(\sigma(a))=\sigma\left(l^{-1}\left(t_{1}, \ldots, t_{s+1}\right)\right) \cdot \sigma(a)
$$

for $\left(t_{1}, \ldots, t_{s+1}\right) \in l\left(\mathcal{O}_{K}^{*+}\right)$. Since $l\left(\mathcal{O}_{K}^{*+}\right)$ and $\sigma\left(\mathcal{O}_{K}\right)$ are lattices of $L$ and $\mathbb{R}^{s} \times \mathbb{C}$ respectively, we have an extension $\bar{\phi}: L \rightarrow \operatorname{Aut}\left(\mathbb{R}^{s} \times \mathbb{C}\right)$ of $\phi$ and $l\left(\mathcal{O}_{K}^{*+}\right) \ltimes_{\phi} \sigma\left(\mathcal{O}_{K}\right)$ can be seen as a lattice of $L \ltimes_{\bar{\phi}}\left(\mathbb{R}^{s} \times \mathbb{C}\right)$. Since we have $\phi\left(t_{1}, \ldots, t_{s+1}\right)=\operatorname{diag}\left(e^{t_{1}}, \ldots, e^{t_{s}}, \sigma_{s+1}\left(l^{-1}\left(t_{1}, \ldots, t_{s+1}\right)\right)\right)$ and $\sigma_{s+1}$ is a complex embedding of $K$, for some $c_{1}, \ldots, c_{s} \in \mathbb{R}$, the Lie group $L \ltimes_{\bar{\phi}}\left(\mathbb{R}^{s} \times \mathbb{C}\right)$ is identified with the Lie group $G$ as above.

Remark 10.2. In $[\mathbf{2 5}]$, for each $s$ Oeljeklaus and Toma constructed a LCK (locally conformal Kähler) structure on the manifold $G / l\left(\mathcal{O}_{K}^{*+}\right) \ltimes_{\phi}$ $\sigma\left(\mathcal{O}_{K}\right)$ and showed that for $s=2$ this LCK manifold is a counter example of Vaisman's conjecture (i.e. every compact LCK manifold has an odd Betti number). By the above proposition, for $s=2 m$ the Betti number $b_{p}=b_{4 m+2-p}={ }_{2 m} C_{p}$ is even for odd number $1 \leq p<2 m$. Hence for any even $s, G / l\left(\mathcal{O}_{K}^{*+}\right) \ltimes_{\phi} \sigma\left(\mathcal{O}_{K}\right)$ is also a counterexample of Vaisman's conjecture.

\section{References}

[1] D. Arapura, Kähler solvmanifolds, Int. Math. Res. Not. 3 (2004), 131-137, MR2038772, Zbl 1091.53047.

[2] D. Arapura, \& M. Nori, Solvable fundamental groups of algebraic varieties and Kähler manifolds, Compositio Math. 116 (1999), no. 2, 173-188, MR1686777, Zbl 0971.14020 .

[3] L. Auslander, An exposition of the structure of solvmanifolds, I. Algebraic theory, Bull. Amer. Math. Soc. 79 (1973), no. 2, 227-261, MR 0486307, Zbl 0265.22016.

[4] O. Baues, Infra-solvmanifolds and rigidity of subgroups in solvable linear algebraic groups, Topology 43 (2004), no. 4, 903-924, MR 2061212, Zbl 1059.57022.

[5] O. Baues, \& V. Cortés, Aspherical Kähler manifolds with solvable fundamental group, Geom. Dedicata 122 (2006), 215-229, MR 2295551, Zbl 1128.53043.

[6] C. Benson \& C. S. Gordon, Kähler and symplectic structures on nilmanifolds, Topology 27 (1988), no. 4, 513-518, MR 0976592, Zbl 0672.53036.

[7] C. Benson \& C.S. Gordon, Kähler structures on compact solvmanifolds, Proc. Amer. Math. Soc. 108 (1990), no. 4, 971-980, MR 0993739, Zbl 0689.53036.

[8] A. Borel, Linear algebraic groups, 2nd ed., Graduate Texts in Mathematics, vol. 126, Springer-Verlag, New York, 1991, MR 1102012, Zbl 0726.20030.

[9] D. Burde, K. Dekimpe \& K. Vercammen, Complete LR-structures on solvable Lie algebras, J. Group Theory 13 (2010), no. 5, 703-719, MR 2720199, Zbl pre05825397.

[10] K. Dekimpe, Solvable Lie algebras, Lie groups and polynomial structures, Compositio Math. 121 (2000), no. 2, 183-204, MR 1757881, Zbl 1002.22003.

[11] P. Deligne, P. Griffiths, J. Morgan \& D. Sullivan, Real homotopy theory of Kähler manifolds, Invent. Math. 29 (1975), no. 3, 245-274, MR 0382702, Zbl 0312.55011. 
[12] N. Dungey, A.F.M. ter Elst \& D.W. Robinson, Analysis on Lie Groups with Polynomial Growth, Progress in Mathematics, 214. Birkhäuser Boston (2003), MR 2000440, Zbl 1041.43003.

[13] Y. Félix, J. Oprea \& D. Tanré, Algebraic Models in Geometry, Oxford Graduate Texts in Mathematics 17, Oxford University Press 2008, MR 2403898, Zbl 1149.53002 .

[14] R.M. Hain, The Hodge de Rham theory of relative Malcev completion, Ann. Sci. Ecole Norm. Sup. (4) 31 (1998), no. 1, 47-92, MR 1604294, Zbl 0911.14008.

[15] K. Hasegawa, Minimal models of nilmanifolds, Proc. Amer. Math. Soc. 106 (1989), no. 1, 65-71, MR 0946638, Zbl 0691.53040.

[16] K. Hasegawa, A class of compact Kählerian solvmanifolds and a general conjecture, Geom. Dedicata 78 (1999), no. 3, 253-258, MR 1725375, Zbl 0940.53037.

[17] K. Hasegawa, A note on compact solvmanifolds with Kähler structures, Osaka J. Math. 43 (2006), no. 1, 131-135, MR 2222405, Zbl 1105.32017.

[18] A. Hattori, Spectral sequence in the de Rham cohomology of fibre bundles, J. Fac. Sci. Univ. Tokyo Sect. I 8 (1960) 289-331, MR 0124918, Zbl 0099.18003.

[19] H. Kasuya, Formality and hard Lefschetz properties of aspherical manifolds, http://arxiv.org/abs/0910.1175, to appear in Osaka J. Math.

[20] G.D. Mostow, Cohomology of topological groups and solvmanifolds, Ann. of Math. (2) 73 (1961), 20-48, MR 0125179, Zbl 0103.26501.

[21] I. Nakamura, Complex parallelisable manifolds and their small deformations, J. Differential Geometry 10 (1975), 85-112, MR 0393580, Zbl 0297.32019.

[22] A. Narkawicz, Cohomology Jumping Loci and Relative Malcev Completion, thesis (Ph.D.)-Duke University, 2007, MR 2711033.

[23] K. Nomizu, On the cohomology of compact homogeneous spaces of nilpotent Lie groups, Ann. of Math. (2) 59 (1954), 531-538, MR 0064057, Zbl 0058.02202.

[24] A.L. Onishchik, E.B. Vinberg (Eds.), Lie groups and Lie algebras. II. Discrete subgroups of Lie groups and cohomologies of Lie groups and Lie algebras, Encyclopaedia of Mathematical Sciences, 21, Springer-Verlag, Berlin (2000), MR 1756406, Zbl 0932.00011.

[25] K. Oeljeklaus, M. Toma, Non-Kähler compact complex manifolds associated to number fields, Ann. Inst. Fourier (Grenoble) 55 (2005), no. 1, 161-171, MR 2141693, Zbl 1071.32017.

[26] M.S. Raghunathan, Discrete subgroups of Lie groups, Springer-Verlag, New York, 1972. Ergebnisse der Mathematik und ihrer Grenzgebiete, Band 68, MR 0507234, Zbl 0254.22005 .

[27] H. Sawai, A construction of lattices on certain solvable Lie groups, Topology Appl. 154 (2007), no. 18, 3125-3134, MR 2364640, Zbl 1138.53065.

[28] C. T. Simpson, Higgs bundles and local systems, Inst. Hautes Études Sci. Publ. Math. No. 75 (1992), 5-95, MR 1179076, Zbl 0814.32003.

[29] D. Sullivan, Infinitesimal computations in topology, Inst. Hautes Études Sci. Publ. Math. No. 47 (1977), 269-331 (1978), MR 0646078, Zbl 0374.57002.

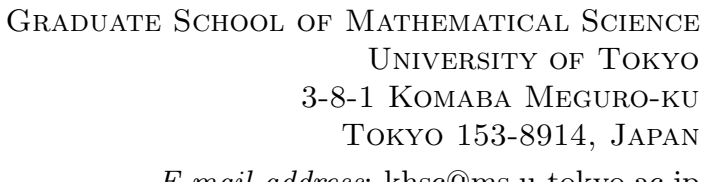

E-mail address: khsc@ms.u-tokyo.ac.jp 